\title{
Adiabatic regularization for Dirac fields in time-varying electric backgrounds
}

\author{
Pau Beltrán-Palau $\odot^{*}$ Jose Navarro-Salas $\odot,{ }^{\dagger}$ and Silvia Pla $\odot^{\ddagger}$ \\ Departamento de Fisica Teorica and IFIC, Centro Mixto Universidad de Valencia-CSIC. Facultad de Fisica, \\ Universidad de Valencia, Burjassot-46100, Valencia, Spain
}

(Received 25 January 2020; revised manuscript received 1 April 2020; accepted 3 May 2020; published 20 May 2020)

\begin{abstract}
The adiabatic regularization method was originally proposed by Parker and Fulling to renormalize the energy-momentum tensor of scalar fields in expanding universes. It can be extended to renormalize the electric current induced by quantized scalar fields in a time-varying electric background. This can be done in a way consistent with gravity if the vector potential is considered as a variable of adiabatic order one. Assuming this, we further extend the method to deal with Dirac fields in four spacetime dimensions. This requires a self-consistent ansatz for the adiabatic expansion, in presence of a prescribed time-dependent electric field, which is different from the conventional expansion used for scalar fields. Our proposal is consistent, in the massless limit, with the conformal anomaly. We also provide evidence that our proposed adiabatic expansion for the fermionic modes parallels the Schwinger-DeWitt adiabatic expansion of the two-point function. We give the renormalized expression of the electric current and analyze, using numerical and analytical tools, the pair production induced by a Sauter-type electric pulse. We also analyze the scaling properties of the current for a large field strength.
\end{abstract}

DOI: 10.1103/PhysRevD.101.105014

\section{INTRODUCTION}

The landmark work of Heisenberg and Euler [1], motivated by earlier work of Sauter [2], established the instability of the quantum vacuum under the influence of a prescribed (slowly varying) electric field. If the field is sufficiently strong, real electron-positron pairs can be created. This result was reobtained by Schwinger in the modern language of quantum electrodynamics by finding a positive imaginary contribution to the effective action $W$. The quantity $e^{-2 \operatorname{Im} W}$ represents then the probability that no actual pair creation occurs during the history of the field [3].

The quantum mechanism driving the spontaneous creation of particles by a gravitational field was discovered by Parker in the early sixties by studying quantized fields in an expanding universe. The crucial fact is as follows [4]: creation and annihilation operators evolve, under the influence of the expansion of the universe (or a generic time-varying gravitational field), into a superposition of creation and annihilation operators. During a cosmic period

\footnotetext{
*pau.beltran@uv.es

jnavarro@ific.uv.es

silvia.pla@uv.es
}

Published by the American Physical Society under the terms of the Creative Commons Attribution 4.0 International license. Further distribution of this work must maintain attribution to the author(s) and the published article's title, journal citation, and DOI. Funded by SCOAP ${ }^{3}$. when the expansion factor is almost constant one can interpret the effect of the gravitational field on the particle number and unambiguously establish the spontaneous creation of real particles by the evolving gravitational field. Major applications of this remarkable phenomena occurs in the very early universe [5,6] and in the vicinity of a collapsing star forming a black hole [7]. These pioneer works on particle creation launched the theory of quantum fields in curved spacetime, as a first step to merge gravity and quantum mechanics within a self-consistent and successful framework [8-11]. The underlying machinery was also employed to study time-varying electromagnetic fields $[12,13]$. In the limit of a slowly varying electric field the Schwinger result can be recovered.

In the gravitational scenario, the most relevant physical observable is the energy-momentum tensor. Its vacuum expectation value $\left\langle T_{\mu \nu}\right\rangle$ possesses ultraviolet (UV) divergences and has to be regularized and renormalized. In the seventies many methods were proposed to this end, as explained in the monographs [8-11]. For homogeneous, time-dependent spacetimes a generic expression for $\left\langle T_{\mu \nu}\right\rangle$ was obtained for scalar fields within the so-called adiabatic regularization scheme [14-18]. The adiabatic method uses a mode by mode subtraction process, naturally suggested by the definition of a single-particle state in an expanding universe, and in such a way that preserves the basic symmetries of the theory. Furthermore, the adiabatic method has been proved to be equivalent to the point-splitting Schwinger-DeWitt renormalization scheme $[15,16,18]$. 
The adiabatic expansion of the field modes parallels the Schwinger-DeWitt adiabatic expansion of the Feynman propagator in Friedmann-Lemaitre-Robertson-Walker (FLRW) spacetimes. The advantage of the adiabatic regularization method is that it very efficient to implement numerical computations, and it is widely used in cosmology. It has been recently extended to spin-1/2 fields in FLRW universes $[19,20]$.

As mentioned above, the analysis of particle creation by time-varying electric fields can be carried out using the techniques first proposed to treat curved backgrounds. The electromagnetic field is considered as an external, unquantized background, while the created particles are excitations of the quantized matter field. From the experimental side, this particle production effect is also of special interest since it may not be far from being experimentally detected in high intensity lasers [21], and in beam-beam collisions [22]. This effect is also very important in astrophysical $[23,24]$ and cosmological scenarios [25-27], and in nonequilibrium processes induced by strong fields [28]. In this context, the most important physical local expectation value is the electric current $\left\langle j_{\mu}\right\rangle$, which also possesses ultraviolet divergences and has to be renormalized in a proper way. Recent discussions on foundational issues related to the particle number density of the created particles, adiabatic invariance, and unitary evolution can be seen in [29-31].

Due to the similarities with the gravitational case, it is a good strategy to readapt the adiabatic regularization scheme to the case in which the external background is an electric field. This program was initiated in $[32,33]$ to study backreaction problems when the matter field is a charged scalar field. It was further extended to treat charged Dirac fermions [34]. It was assumed that the adiabatic order of the vector potential $A_{\mu}$ is 0 . The problem was reconsidered for a charged scalar field in [35] by assuming that the adiabatic order of $A_{\mu}$ is 1 , instead of zero. This new reassignment of the adiabatic order for $A_{\mu}$ is an unavoidable requirement in presence of a gravitational background. The argument was reinforced in $[36,37]$ on the basis of the covariant conservation of the energy-momentum tensor. The adiabatic regularization of two-dimensional fermions incorporating the adiabatic order assignment 1 for $A_{\mu}$ has been further reanalyzed in $[31,35,38]$. Other renormalization methods have been generalized to incorporate an electromagnetic background, as for instance the Hadamard point-splitting method for complex scalar fields $[39,40]$, with results in agreement with [35].

Within the above context, it seems natural to extend the adiabatic regularization/renormalization method, with the assumption that $A_{\mu}$ is of adiabatic order 1, to Dirac fields in presence of an electric field background in four spacetime dimensions. This is the main aim of this work. As stressed above, previous studies in the literature on this problem [34] assumed that $A_{\mu}$ is of adiabatic order 0 . This extension requires a self-consistent ansatz for the adiabatic expansion of the field modes. We give a proper ansatz, which cannot be fitted within the Wentzel-Kramers-Brillouin (WKB)type expansion used for scalar fields [8,10,11]. Our extension of the adiabatic method is in agreement with the trace anomaly. Even more, we provide strong evidence that our adiabatic expansion of the field modes parallels the adiabatic Schwinger-DeWitt expansion of the propagator. In addition to the trace anomaly, our adiabatic expansion also reproduces the DeWitt coefficient $E_{3}$, at sixth adiabatic order. We carry out the adiabatic renormalization and provide a general expression for the renormalized electric current. We illustrate the power of the method by studying with detail a Sauter-type electric pulse.

The paper is organized as follows. In Sec. II we will describe the status of adiabatic regularization when a timevarying electric field is part of the background. We will give strong reasons for adopting a new viewpoint and reprehend the problem of the adiabatic regularization of charged $4 d$ fermions in time-dependent electric fields. In Sec. III we introduce the basic ingredients of our ansatz to construct the adiabatic expansion of the four-dimensional fermionic modes coupled to a prescribed time-dependent electric field. Section IV is devoted to explain the details of the adiabatic renormalization procedure in this context. In particular, we give a generic and explicit expression of the renormalized electric current. We also test the consistency of the method and discuss some intrinsic renormalization ambiguities. In Sec. V we study the particular case in which the background field is a Sauter-type electric pulse. We analyze the particle production phenomena in terms of the renormalized electric current. We also discuss the scaling properties of the created current. In Sec. VI we state our main conclusions. Our work is complemented with a series of appendices where we give technical details. We also discuss in the Appendix B the connection between the adiabatic method and the Hadamard renormalization scheme for charged scalar fields.

\section{BACKGROUND AND MOTIVATION}

To motivate the main idea of this work it is very convenient to present the status of the adiabatic regularization method for a charged 4-dimensional scalar field interacting with a classical, homogeneous, time-dependent electric background. We will assume that the electric field is of the form $\vec{E}=(0,0, E(t))$ with potential vector $A_{\mu}=(0,0,0,-A(t))$. We will also assume that the spacetime is described by a FLRW metric of the form $d s^{2}=d t^{2}-a^{2}(t) d \vec{x}^{2}$. The Klein Gordon equation reads

$$
\left(D_{\mu} D^{\mu}+m^{2}+\xi R\right) \phi=0,
$$

where $D_{\mu} \phi=\left(\nabla_{\mu}+i q A_{\mu}\right) \phi$ and $R$ is the Ricci scalar. Since the potential vector $A_{\mu}$ is homogeneous, one 
can expand the scalar field in modes as $\phi=$ $\frac{1}{\sqrt{2(2 \pi a)^{3}}} \int d^{3} k\left(A_{\vec{k}} e^{i \vec{k} \vec{x}} h_{\vec{k}}+B_{\vec{k}} e^{-i \vec{k} \vec{x}} h_{-\vec{k}}^{*}\right)$, where the mode functions $h_{\vec{k}}(t)$ satisfy

$$
\ddot{h}_{\vec{k}}+\left(a^{-2}\left(k_{3}+q A\right)^{2}+a^{-2} k_{\perp}^{2}+m^{2}+\sigma\right) h_{\vec{k}}=0,
$$

with $\sigma=(6 \xi-3 / 4) \dot{a}^{2} / a^{2}+(6 \xi-3 / 2) \ddot{a} / a$. Once we have obtained the mode equation (2), we can make an adiabatic expansion of the field modes. To this end, one can propose the usual WKB ansatz

$$
h_{\vec{k}}=\frac{1}{\sqrt{\Omega_{\vec{k}}}} e^{-i \int \Omega_{\vec{k}}(t) d t},
$$

where $W_{k}$ can be expanded adiabatically, in powers of derivatives of $a(t)$ and $A(t)$, as $\Omega_{\vec{k}}=\sum_{n=0}^{\infty} \omega^{(n)}$.

The choice of the leading terms $\omega^{(0)}$ is a crucial ingredient to define the adiabatic expansion. For $A=0$ the proper choice for $\omega^{(0)}$ is $\omega^{(0)}=\omega=\sqrt{\vec{k}^{2} / a^{2}+m^{2}}$. This defines the conventional adiabatic expansion for a scalar field, as first introduced in the pioneer works [14]. When the background spacetime is Minkowski $a=1$, the choice proposed in [32] was $\omega^{(0)}=\omega=\sqrt{(\vec{k}-q \vec{A})^{2}+m^{2}}$. This choice assumes that $A(t)$ should be treated as a variable of zero adiabatic order, like $a(t)$. As noted in [35,36], this choice runs into difficulties in presence of a gravitational background. It was proposed in $[35,36]$ that the leading term should be maintained as $\omega^{(0)}=\omega=\sqrt{\vec{k}^{2} / a^{2}+m^{2}}$, even in the presence of an electromagnetic field. This means that $A(t)$ must be considered as a variable of adiabatic order 1 , like $\dot{a}$. Hence $\dot{A}$ is of adiabatic order 2, etc.

Next to leading order terms can be obtained recursively from (2). The adiabatic expansion allows us to regularize the observables performing adiabatic subtractions. Since the first terms of the adiabatic expansion capture all potential ultraviolet divergences, one can subtract them, obtaining finite and meaningful results. With this method, we obtain the following vacuum expectation value of the two point function

$$
\left\langle\phi^{\dagger} \phi\right\rangle_{\mathrm{ren}}=\frac{1}{2(2 \pi a(t))^{3}} \int d^{3} k\left[\left|h_{\vec{k}}\right|^{2}-\left\langle\phi^{\dagger} \phi\right\rangle_{\vec{k}}^{(0-2)}\right],
$$

where $\left\langle\phi^{\dagger} \phi\right\rangle_{\vec{k}}^{(0-2)}=\sum_{n=0}^{2}\left(\Omega_{\vec{k}}^{-1}\right)^{(n)}$.
As stressed in the introduction, the adiabatic expansion of the field modes translates into an adiabatic expansion of the two-point function. The latter turns out to be equivalent to the Schwinger-DeWitt expansion of the Feynman propagator $\left\langle T \phi^{\dagger}(x) \phi\left(x^{\prime}\right)\right\rangle$. We will show this explicitly at the coincident limit $x^{\prime} \rightarrow x$ at fourth and sixth adiabatic orders. At fourth adiabatic order the corresponding momentum integral is finite and we get

$$
\begin{aligned}
\left\langle\phi^{\dagger} \phi\right\rangle^{(4)}= & \frac{1}{2(2 \pi a)^{3}} \int d^{3} k\left\langle\phi^{\dagger} \phi\right\rangle_{\vec{k}}^{(4)} \\
= & \frac{1}{16 \pi^{2} m^{2}}\left(\frac{36 \dot{a}^{2} \xi^{2} \ddot{a}}{a^{3}}-\frac{17 \dot{a}^{2} \xi \ddot{a}}{a^{3}}+\frac{29 \dot{a}^{2} \ddot{a}}{15 a^{3}}\right. \\
& +\frac{18 \xi^{2} \ddot{a}^{2}}{a^{2}}-\frac{5 \xi \ddot{a}^{2}}{a^{2}}+\frac{3 \ddot{a}^{2}}{10 a^{2}}+\frac{18 \dot{a}^{4} \xi^{2}}{a^{4}}-\frac{6 \dot{a}^{4} \xi}{a^{4}} \\
& \left.+\frac{a^{(4)} \xi}{a}+\frac{\dot{a}^{4}}{2 a^{4}}-\frac{a^{(4)}}{5 a}+\frac{\dot{A}^{2} q^{2}}{6 a^{2}}+\frac{3 a^{(3)} \dot{a} \xi}{a^{2}}-\frac{3 a^{(3)} \dot{a}}{5 a^{2}}\right) .
\end{aligned}
$$

It is not difficult to check that the above result can be reexpressed in the following covariant form

$$
\left\langle\phi^{\dagger} \phi\right\rangle^{(4)}=\frac{E_{2}}{16 \pi^{2} m^{2}},
$$

where $E_{2}$ matches exactly the DeWitt coefficient [41]

$$
\begin{aligned}
E_{2}= & -\frac{1}{30} \square R+\frac{1}{72} R^{2}-\frac{1}{180} R^{\mu \nu} R_{\mu \nu}+\frac{1}{180} R^{\mu \nu \rho \sigma} R_{\mu \nu \rho \sigma} \\
& -\frac{q^{2}}{12} F^{\mu \nu} F_{\mu \nu}+\frac{1}{2} Q^{2}-\frac{1}{6} R Q+\frac{1}{6} \square Q .
\end{aligned}
$$

In the above expression $Q$ is given by $Q=\xi R$. We note that, to obtain equivalence with the Schwinger-DeWitt proper-time method it has been essential to assume that $A_{\mu}$ is of adiabatic order $1, F_{\mu \nu}$ of adiabatic order 2, etc. With the zero adiabatic order assignment for $A_{\mu}$ one obtains a noncovariant and ill-defined expression for $\left\langle\phi^{\dagger} \phi\right\rangle^{(4)}$. With our proposed leading order choice for $\omega^{(0)}=\omega=$ $\sqrt{\vec{k}^{2} / a^{2}+m^{2}}$ we find equivalence with the SchwingerDeWitt expansion at very nontrivial higher orders. For instance, our calculation for $\left\langle\phi^{\dagger} \phi\right\rangle^{(6)}$ gives 


$$
\begin{aligned}
\left\langle\phi^{\dagger} \phi\right\rangle^{(6)}= & \frac{1}{2(2 \pi a)^{3}} \int d^{3} k\left\langle\phi^{\dagger} \phi\right\rangle_{\vec{k}}^{(6)} \\
= & -\frac{108 \dot{a}^{4} \xi^{3} \ddot{a}}{a^{5}}+\frac{96 \dot{a}^{4} \xi^{2} \ddot{a}}{a^{5}}-\frac{217 \dot{a}^{4} \xi \ddot{a}}{10 a^{5}}+\frac{197 \dot{a}^{4} \ddot{a}}{140 a^{5}}-\frac{108 \dot{a}^{2} \xi^{3} \ddot{a}^{2}}{a^{4}}+\frac{75 \dot{a}^{2} \xi^{2} \ddot{a}^{2}}{a^{4}}-\frac{221 \dot{a}^{2} \xi \ddot{a}^{2}}{10 a^{4}}+\frac{159 \dot{a}^{2} \ddot{a}^{2}}{70 a^{4}}-\frac{\dot{A}^{2} \xi q^{2} \ddot{a}}{a^{3}} \\
& +\frac{\dot{a} \dot{A} q^{2} \ddot{a}}{90 a^{3}}+\frac{23 \dot{A}^{2} q^{2} \ddot{a}}{90 a^{3}}-\frac{36 \xi^{3} \ddot{a}^{3}}{a^{3}}-\frac{24 a^{(3)} \dot{a} \xi^{2} \ddot{a}}{a^{3}}+\frac{12 \xi^{2} \ddot{a}^{3}}{a^{3}}+\frac{133 a^{(3)} \dot{a} \xi \ddot{a}}{10 a^{3}}+\frac{3 \xi \ddot{a}^{3}}{5 a^{3}}-\frac{103 a^{(3)} \dot{a} \ddot{a}}{60 a^{3}}-\frac{14 \ddot{a}^{3}}{45 a^{3}}-\frac{q^{2} \ddot{a}^{2}}{20 a^{2}} \\
& -\frac{6 a^{(4)} \xi^{2} \ddot{a}}{a^{2}}+\frac{17 a^{(4)} \xi \ddot{a}}{10 a^{2}}-\frac{43 a^{(4)} \ddot{a}}{420 a^{2}}-\frac{36 \dot{a}^{6} \xi^{3}}{a^{6}}+\frac{6 \dot{a}^{6} \xi^{2}}{a^{6}}+\frac{\dot{a}^{6} \xi}{a^{6}}-\frac{a^{(6)} \xi}{10 a}-\frac{\dot{a}^{6}}{6 a^{6}}+\frac{3 a^{(6)}}{140 a}-\frac{\dot{a}^{2} \dot{A}^{2} \xi q^{2}}{a^{4}}+\frac{37 \dot{a}^{2} \dot{A}^{2} q^{2}}{180 a^{4}} \\
& -\frac{\dot{A} A^{(3)} q^{2}}{15 a^{2}}-\frac{2 a^{(5)} \dot{a} \xi}{5 a^{2}}+\frac{3 a^{(5)} \dot{a}}{35 a^{2}}-\frac{6 a^{(3)} \dot{a}^{3} \xi^{2}}{a^{4}}-\frac{6 a^{(4)} \dot{a}^{2} \xi^{2}}{a^{3}}+\frac{7 a^{(3)} \dot{a}^{3} \xi}{10 a^{4}}+\frac{31 a^{(4)} \dot{a}^{2} \xi}{10 a^{3}}+\frac{13 a^{(3)} \dot{a}^{3}}{140 a^{4}}-\frac{23 a^{(4)} \dot{a}^{2}}{60 a^{3}} \\
& -\frac{3\left(a^{(3)}\right)^{2} \xi^{2}}{a^{2}}+\frac{7\left(a^{(3)}\right)^{2} \xi}{10 a^{2}}-\frac{\left(a^{(3)}\right)^{2}}{42 a^{2}},
\end{aligned}
$$

where $a^{(n)}$ refers to $d^{n} a / d t^{n}$. The result turns out to be proportional to the corresponding DeWitt coefficient of sixth adiabatic order $E_{3}[42,43]$. The covariant expression is given in the Appendix A.

We stress again that it has been crucial for obtaining the above results the choice $\omega^{(0)}=\omega=\sqrt{\vec{k}^{2} / a^{2}+m^{2}}$, instead of $\omega^{(0)}=\omega=\sqrt{(\vec{k}-q \vec{A})^{2} / a^{2}+m^{2}}$. For completeness, a comparison of the above formulation of the adiabatic regularization with the Hadamard renormalization scheme is given in the Appendix B.

\section{A. Adiabatic regularization for fermions in two-dimensions}

To reinforce the previous analysis, and prior to face the adiabatic regularization of charged fermions in four spacetime dimensions, it is also convenient to consider the problem for a charged Dirac field in two-dimensions. We will follow $[35,36]$ and compare the results with the pioneer analysis in [34]. The comparison will allow us to understand why it has been necessary to reprehend the problem, as already stressed above.

The quantum field satisfies the Dirac equation $\left(i \underline{\gamma}^{\mu} D_{\mu}-m\right) \psi=0$, where $D_{\mu} \equiv \nabla_{\mu}-\Gamma_{\mu}-i q A_{\mu}$ and $\Gamma_{\mu}$ is the spin connection. The curved space Dirac matrices satisfy the anticommutation relations $\left\{\underline{\gamma}^{\mu}, \underline{\gamma}^{\mu}\right\}=2 g^{\mu \nu}$. We assume a homogeneous, time-dependent electric background $E(t)$, with associated potential vector $A_{\mu}=$ $(0,-A(t))$. The metric is also assumed of the FLRW form $d s^{2}=d t^{2}-a^{2}(t) d x^{2}$. One can expand the Dirac field as $\psi=\int_{-\infty}^{\infty} d k\left[B_{k} u_{k}(t, x)+D^{\dagger} v_{k}(x, t)\right]$, where the two independent spinor solutions can be written as

$u_{k}(t, x)=\frac{e^{i k x}}{\sqrt{2 \pi a}}\left(\begin{array}{c}h_{k}^{I}(t) \\ -h_{k}^{I I}(t)\end{array}\right), \quad v_{k}(t, x)=\frac{e^{-i k x}}{\sqrt{2 \pi a}}\left(\begin{array}{c}h_{-k}^{I I *}(t) \\ h_{-k}^{I *}(t)\end{array}\right)$.
The classical electric field satisfies the semiclassical Maxwell equations $\nabla_{\mu} F^{\mu \nu}=-q\left\langle\bar{\psi} \gamma \gamma^{\nu} \psi\right\rangle_{\text {ren }}=\left\langle j^{\nu}\right\rangle_{\text {ren }}$, which in our system turns out to be a single equation $\dot{E}=-\left\langle j^{x}\right\rangle_{\text {ren }}$. In this scenario the adiabatic rules are univocally fixed: $a(t)$ has to be considered of adiabatic order 0 , the energy-momentum tensor must be regularized up to the second adiabatic order and the electric current must to be regularized up to the first adiabatic order. The adiabatic subtractions required to regularize the electric current $\left\langle j^{x}\right\rangle$ will be different depending on the adiabatic order that we choose for the background field $A(t)$, i.e.,

$$
\begin{aligned}
\left\langle j^{x}\right\rangle_{\mathrm{ren}}^{A \sim O(0)}= & q \int \frac{d k}{2 \pi a}\left(\left|h_{k}^{I I}\right|^{2}-\left|h_{k}^{I}\right|^{2}\right. \\
& \left.-\frac{k+q A}{a \sqrt{(k+q A)^{2} / a^{2}+m^{2}}}\right), \\
\left\langle j^{x}\right\rangle_{\mathrm{ren}}^{A \sim O(1)}= & q \int \frac{d k}{2 \pi a}\left(\left|h_{k}^{I I}\right|^{2}-\left|h_{k}^{I}\right|^{2}-\frac{k}{a \omega}-\frac{m^{2} q A}{a \omega^{3}}\right),
\end{aligned}
$$

where $\omega=\sqrt{k^{2} / a^{2}+m^{2}}$. In (10) we have considered $A$ of adiabatic order zero, while in (11) we have considered it of adiabatic order one. One can check that the subtractions obtained in the first case are the same to the ones obtained in [34] for $a=1$. Although it can be proven [37] that these two choices are equivalent when $a=1$, in the sense that $\triangle\left\langle j^{x}\right\rangle_{\mathrm{ren}}=\left\langle j^{x}\right\rangle_{\mathrm{ren}}^{A \sim O(0)}-\left\langle j^{x}\right\rangle_{\mathrm{ren}}^{A \sim O(1)}=0$, they are in general nonequivalent. We can see how gravity breaks this equivalence. In the second case we can easily see that the energy density is covariantly conserved

$$
\nabla_{\mu}\left\langle T^{\mu 0}\right\rangle_{\text {ren }}+\nabla_{\mu} T_{\text {elec }}^{\mu 0}=E\left(\dot{E}+\left\langle j^{x}\right\rangle_{\text {ren }}\right)=0 .
$$

But, when we consider $A(t)$ of adiabatic order 0 , the conservation does not hold any more, and one finds $\nabla_{\mu}\left\langle T^{\mu 0}\right\rangle_{\text {ren }}+\nabla_{\mu} T_{\text {elec }}^{\mu 0} \sim E(t)\left\langle j^{x}\right\rangle^{(2)}$, where $\left\langle j^{x}\right\rangle^{(2)}$ is the 
subtraction term of adiabatic order two, which cannot be properly absorbed into the definition of the electric current.

Moreover, only when $A$ is considered of adiabatic order 1 the adiabatic expansion of the field modes turns out to be equivalent to the Schwinger-DeWitt expansion of the twopoint function. For instance, the adiabatic expansion of the two-point function at coincidence is found to be (at second and fourth adiabatic order)

$$
\begin{aligned}
\langle\bar{\psi} \psi\rangle^{(2)} & =\frac{1}{4 \pi m}\left(\frac{\ddot{a}}{3 a}\right)=-\frac{\operatorname{tr} E_{1}}{4 \pi m}, \\
\langle\bar{\psi} \psi\rangle^{(4)} & =\frac{1}{4 \pi m^{3}}\left(-\frac{\dot{a}^{2} \ddot{a}}{30 a^{3}}+\frac{\ddot{a}^{2}}{15 a^{2}}-\frac{a^{(4)}}{30 a}+\frac{2 \dot{A}^{2} q^{2}}{3 a^{2}}+\frac{a^{(3)} \dot{a}}{30 a^{2}}\right) \\
& =-\frac{\operatorname{tr} E_{2}}{4 \pi m^{3}},
\end{aligned}
$$

where $E_{1}$ and $E_{2}$ are the corresponding DeWitt coefficients. They are given, in the covariant form, by $[8,42,43]$

$$
\begin{aligned}
E_{1}= & \frac{1}{6} R I-Q \\
E_{2}= & \left(-\frac{1}{30} \square R+\frac{1}{72} R^{2}-\frac{1}{180} R^{\mu \nu} R_{\mu \nu}+\frac{1}{180} R^{\mu \nu \rho \sigma} R_{\mu \nu \rho \sigma}\right) I \\
& +\frac{1}{12} W^{\mu \nu} W_{\mu \nu}+\frac{1}{2} Q^{2}-\frac{1}{6} R Q+\frac{1}{6} \square Q
\end{aligned}
$$

where $Q=\frac{1}{4} R I-\frac{i}{2} q F_{\mu \nu} \underline{\gamma}^{\mu} \underline{\gamma}^{\nu}$ and $W_{\mu \nu}=-i q F_{\mu \nu} I-\frac{1}{4} R_{\mu \nu \rho \sigma} \underline{\gamma^{\rho}} \underline{\gamma}^{\sigma}$.

The above arguments make it necessary to reconsider the problem of adiabatic regularization for fermions in timevarying electric backgrounds in four dimensions. We will adopt the view of considering $A_{\mu}$ of adiabatic order 1, as advocated in $[31,35,36,38]$, and in contrast to the view adopted in [34]. The main reasons, as exposed above, are (i) expected agreement with the Schwinger-DeWitt adiabatic expansion of the two-point function at coincidence; (ii) consistency with the covariant conservation of the energy-momentum tensor when gravity is turned on. We think these are convincing arguments to go further with our proposed approach. For simplicity we will restrict our analysis to Minkowski spacetime.

\section{4D DIRAC FIELDS: MODE EQUATIONS, ANSATZ AND ADIABATIC EXPANSION}

Let us consider a massive 4-dimensional spinor field $\psi$ interacting with a prescribed electric field. The corresponding Dirac equation reads

$$
\left(i \gamma^{\mu} D_{\mu}-m\right) \psi=0
$$

where $D_{\mu} \equiv \partial_{\mu}-i q A_{\mu}$ and $\gamma^{\mu}$ are the (flat-space) Dirac matrices satisfying the anticommutation relations $\left\{\gamma^{\mu}, \gamma^{\nu}\right\}=$ $2 \eta^{\mu \nu}$. We consider $\psi$ as a quantized Dirac field, while the electromagnetic field is assumed to be a classical and spatially homogeneous field $\vec{E}(t)=(0,0, E(t))$. It is very convenient to choose a gauge such that only the $z$-component of the vector potential is nonvanishing: $A_{\mu}=(0,0,0,-A(t))$, where $E(t)=-\dot{A}(t)$.

To prepare things to propose a consistent ansatz for the adiabatic expansion of the field modes it is very important to transform the Dirac field as $\psi^{\prime}=U \psi$, where $U$ is the unitary operator $U=\frac{1}{\sqrt{2}} \gamma^{0}\left(I-\gamma^{3}\right)$, which verifies $U=U^{\dagger}=U^{-1}$. This transformation will allow us to express the Dirac field in terms of only two time-dependent functions [see (20)]. The field $\psi^{\prime}$ obeys the Dirac equation for the transformed matrices $\gamma^{\prime \mu}=U \gamma^{\mu} U^{\dagger}$, namely: $\gamma^{\prime 0}=$ $\gamma^{3} \gamma^{0}, \gamma^{\prime 1}=-\gamma^{3} \gamma^{1}, \gamma^{\prime 2}=-\gamma^{3} \gamma^{2}, \gamma^{\prime 3}=-\gamma^{3}$. Substituting them in the Dirac equation we easily get

$\left[\gamma^{0} \partial_{0}-\gamma^{1} \partial_{1}-\gamma^{2} \partial_{2}-\partial_{3}-i q A(t)-i m \gamma^{3}\right] \psi^{\prime}=0$.

Expanding the field in Fourier modes, $\psi^{\prime}(t, \vec{x})=$ $\int \frac{d^{3} \vec{k}}{(2 \pi)^{3 / 2}} \psi_{\vec{k}}^{\prime}(t) e^{i \vec{k} \vec{x}}$, we obtain the following equation

$$
\left[\partial_{0}-i \gamma^{0}\left(k_{1} \gamma^{1}+k_{2} \gamma^{2}+m \gamma^{3}\right)-i\left(k_{3}+q A(t)\right) \gamma^{0}\right] \psi_{\vec{k}}^{\prime}(t)=0,
$$

where $\vec{k} \equiv\left(k_{1}, k_{2}, k_{3}\right)$. The form of the above equation allows us to reexpress the field in terms of two-component spinors as follows

$$
\psi_{\vec{k}, \lambda}^{\prime}(t)=\left(\begin{array}{c}
h_{\vec{k}}^{I}(t) \eta_{\lambda}(\vec{k}) \\
h_{\vec{k}}^{I I}(t) \lambda \eta_{\lambda}(\vec{k})
\end{array}\right),
$$

where $\eta_{\lambda}$ with $\lambda= \pm 1$ form an orthonormal basis of two-spinors $\left(\eta_{\lambda}^{\dagger} \eta_{\lambda^{\prime}}=\delta_{\lambda, \lambda^{\prime}}\right)$ verifying $\frac{k_{1} \sigma^{1}+k_{2} \sigma^{2}+m \sigma^{3}}{\sqrt{k_{1}^{2}+k_{2}^{2}+m^{2}}} \eta_{\lambda}=\lambda \eta_{\lambda}$. Their explicit expressions are

$$
\begin{aligned}
& \eta_{+1}(\vec{k})=\frac{1}{\sqrt{2 \kappa(\kappa+m)}}\left(\begin{array}{c}
\kappa+m \\
k_{1}+i k_{2}
\end{array}\right), \\
& \eta_{-1}(\vec{k})=\frac{1}{\sqrt{2 \kappa(\kappa+m)}}\left(\begin{array}{c}
-k_{1}+i k_{2} \\
\kappa+m
\end{array}\right),
\end{aligned}
$$

where $\kappa \equiv \sqrt{k_{1}^{2}+k_{2}^{2}+m^{2}}$. Substituting (20) in (19) and using the Dirac representation for the matrices $\gamma^{\mu}$, one obtains the following differential equations for the functions $h_{\vec{k}}^{I}$ and $h_{\vec{k}}^{I I}$

$$
\begin{aligned}
& \dot{h}_{\vec{k}}^{I}-i\left(k_{3}+q A\right) h_{\vec{k}}^{I}-i \kappa h_{\vec{k}}^{I I}=0, \\
& \dot{h}_{\vec{k}}^{I I}+i\left(k_{3}+q A\right) h_{\vec{k}}^{I I}-i \kappa h_{\vec{k}}^{I}=0 .
\end{aligned}
$$

These equations are exactly the same as those obtained in the two-dimensional case [35], where $\kappa$ plays here the role 
of the mass. With the solutions of these equations we can construct the $u$-type field modes (assumed to be of positive frequency at early times) as follows

$$
u_{\vec{k}, \lambda}(x)=\frac{e^{i \vec{k} \vec{x}}}{(2 \pi)^{3 / 2}}\left(\begin{array}{c}
h_{\vec{k}}^{I}(t) \eta_{\lambda}(\vec{k}) \\
h_{\vec{k}}^{I I}(t) \lambda \eta_{\lambda}(\vec{k})
\end{array}\right) .
$$

Similarly, one can construct the orthogonal $v$-type field modes (of negative frequency at early times) as

$$
v_{\vec{k}, \lambda}(x)=\frac{e^{-i \vec{k} \vec{x}}}{(2 \pi)^{3 / 2}}\left(\begin{array}{c}
-h_{-\vec{k}}^{I I *}(t) \eta_{-\lambda}(-\vec{k}) \\
-h_{-\vec{k}}^{I *}(t) \lambda \eta_{-\lambda}(-\vec{k})
\end{array}\right) .
$$

The normalization conditions for this set of spinors, $\left(u_{\vec{k}, \lambda}, v_{\vec{k}, \lambda^{\prime}}\right)=0, \quad\left(u_{\vec{k}, \lambda}, u_{\vec{k}, \lambda^{\prime}}\right)=\left(v_{\vec{k}, \lambda}, v_{\vec{k}, \lambda^{\prime}}\right)=\delta^{(3)}\left(\vec{k}-\vec{k}^{\prime}\right) \delta_{\lambda \lambda^{\prime}}$, where (,) is the Dirac inner product, are ensured with the normalization condition

$$
\left|h_{\vec{k}}^{I}\right|^{2}+\left|h_{\vec{k}}^{I I}\right|^{2}=1
$$

which will be preserved on time. With this set of basic spinor solutions one can construct the Fourier expansion of the Dirac field operator

$$
\psi^{\prime}(x)=\sum_{\lambda} \int d^{3} \vec{k}\left[B_{\vec{k}, \lambda} u_{\vec{k}, \lambda}(x)+D_{\vec{k}, \lambda}^{\dagger} v_{\vec{k}, \lambda}(x)\right],
$$

where $B_{\vec{k} \lambda}$ and $D_{\vec{k} \lambda}$ are the annihilation operators for particles and antiparticles respectively. The normalization condition (26) guaranties the usual anticommutation relations for these operators: $\left\{B_{\vec{k}, \lambda}, B_{\vec{k}^{\prime}, \lambda^{\prime}}^{\dagger}\right\}=\left\{D_{\vec{k}, \lambda}, D_{\vec{k}^{\prime}, \lambda^{\prime}}^{\dagger}\right\}=$ $\delta^{3}\left(\vec{k}-\overrightarrow{k^{\prime}}\right) \delta_{\lambda, \lambda^{\prime}}$, and all other combinations are 0 .

\section{A. Adiabatic expansion}

Armed with the above results we can determine a consistent adiabatic expansion of the four dimensional Dirac field modes interacting with the prescribed electric background. Based on the two dimensional expansion given in [35], and taking into account that the positivefrequency solution with vanishing electric field, in the representation associated to $\psi^{\prime}$, is given by

$$
\begin{aligned}
h_{\vec{k}}^{I(0)} & =\sqrt{\frac{\omega-k_{3}}{2 \omega}} e^{-i \omega t}, \\
h_{\vec{k}}^{I I(0)} & =-\sqrt{\frac{\omega+k_{3}}{2 \omega}} e^{-i \omega t},
\end{aligned}
$$

with $\omega=\sqrt{k_{3}^{2}+\kappa^{2}}$, we propose the following ansatz for the field modes:

$$
\begin{aligned}
h_{\vec{k}}^{I} & =\sqrt{\frac{\omega-k_{3}}{2 \omega}} F(t) e^{-i \int^{t} \Omega\left(t^{\prime}\right) d t^{\prime}}, \\
h_{\vec{k}}^{I I} & =-\sqrt{\frac{\omega+k_{3}}{2 \omega}} G(t) e^{-i \int^{t} \Omega\left(t^{\prime}\right) d t^{\prime}},
\end{aligned}
$$

where the complex functions $F(t)$ and $G(t)$ and the real function $\Omega(t)$ are expanded adiabatically

$$
\begin{aligned}
& \Omega(t)=\sum_{n=0}^{\infty} \omega^{(n)}(t), \quad F(t)=\sum_{n=0}^{\infty} F^{(n)}(t), \\
& G(t)=\sum_{n=0}^{\infty} G^{(n)}(t) .
\end{aligned}
$$

Here, $\Omega^{(n)}, F^{(n)}$ and $G^{(n)}$ are functions of adiabatic order $n$. The adiabatic order of a given function will be determined by its dependence on the potential vector $A(t)$ and its derivatives. In order to recover at leading order the exact solution with vanishing electric field $A(t)=0$ we demand $F^{(0)}=G^{(0)}=1$ and $\omega^{(0)}=\omega$. With this condition we are implicitly fixing the adiabatic order of the potential vector $A(t)$ to 1 , hence, $\dot{A}(t)$ and $A(t)^{2}$ will be of order $2, \ddot{A}(t)$, $A(t) \dot{A}(t)$ and $A(t)^{3}$ of order three and so on. For a detailed discussion on the adiabatic order assignment see [36].

Plugging the ansatz (30) in the mode equations (22) and (23) and also in the normalization condition (26) we get a system of equations for the functions $F(t), G(t)$ and $\Omega(t)$

$$
\begin{aligned}
\left(\omega-k_{3}\right)\left(\dot{F}-i \Omega F-i\left(k_{3}+q A\right) F\right)+i \kappa^{2} G & =0, \\
\left(\omega+k_{3}\right)\left(\dot{G}-i \Omega G+i\left(k_{3}+q A\right) G\right)+i \kappa^{2} F & =0, \\
\frac{\omega-k_{3}}{2 \omega}|F|^{2}+\frac{\omega+k_{3}}{2 \omega}|G|^{2} & =1 .
\end{aligned}
$$

In order to obtain the expressions of the adiabatic terms $\omega^{(n)}, F^{(n)}$ and $G^{(n)}$, we introduce the expansion (31) into Eqs. (32), (33) and (34) and solve them recursively, order by order. Note that $G\left(k_{3}, q A\right)$ satisfies the same equations as $F\left(-k_{3},-q A\right)$, hence we take $G\left(k_{3}, q A\right)=F\left(-k_{3},-q A\right)$. The system can be solved algebraically by iteration and the general solution is given by

$$
\begin{aligned}
\omega^{(n)}= & \frac{\left(\omega-k_{3}\right)}{2 \omega}\left[\dot{F}_{y}^{(n-1)}-\sum_{i=1}^{n-1} \omega^{(n-i)} F_{x}^{(i)}-q A F_{x}^{(n-1)}\right] \\
& +\frac{\left(\omega+k_{3}\right)}{2 \omega}\left[\dot{G}_{y}^{(n-1)}-\sum_{i=1}^{n-1} \omega^{(n-i)} G_{x}^{(i)}+q A G_{x}^{(n-1)}\right],
\end{aligned}
$$




$$
\begin{aligned}
F_{x}^{(n)}= & \frac{\left(\omega+k_{3}\right)}{4 \omega^{2}}\left[\dot{F}_{y}^{(n-1)}-\sum_{i=1}^{n-1} \omega^{(n-i)} F_{x}^{(i)}-q A F_{x}^{(n-1)}\right. \\
& \left.-\dot{G}_{y}^{(n-1)}+\sum_{i=1}^{n-1} \omega^{(n-i)} G_{x}^{(i)}-q A G_{x}^{(n-1)}\right] \\
& -\frac{\left(\omega-k_{3}\right)}{4 \omega} \sum_{i=1}^{n-1}\left(F_{x}^{(i)} F_{x}^{(n-i)}+F_{y}^{(i)} F_{y}^{(n-i)}\right) \\
& -\frac{\left(\omega+k_{3}\right)}{4 \omega} \sum_{i=1}^{n-1}\left(G_{x}^{(i)} G_{x}^{(n-i)}+G_{y}^{(i)} G_{y}^{(n-i)}\right),
\end{aligned}
$$

$F_{y}^{(n)}=G_{y}^{(n)}-\frac{\left(\omega-k_{3}\right)}{\kappa^{2}}\left[\dot{F}_{x}^{(n-1)}+\sum_{i=1}^{n-1} \omega^{(n-i)} F_{y}^{(i)}+q A F_{y}^{(n-1)}\right]$,

where we have parametrized $F$ and $G$ in terms of real functions as $F=F_{x}+i F_{y}$ and $G=G_{x}+i G_{y}$. Note that there is an ambiguity in the imaginary part (37). However, it disappears when computing physical observables. Further discussions on this issue are given in [20]. For simplicity we choose

$$
\begin{aligned}
F_{y}^{(n)} & =-G_{y}^{(n)} \\
& =-\frac{\left(\omega-k_{3}\right)}{2 \kappa^{2}}\left[\dot{F}_{x}^{(n-1)}+\sum_{i=1}^{n-1} \omega^{(n-i)} F_{y}^{(i)}+q A F_{y}^{(n-1)}\right] .
\end{aligned}
$$

With the initial conditions $F_{x}^{(0)}=G_{x}^{(0)}=1, F_{y}^{(0)}=G_{y}^{(0)}=0$ and $\omega^{(0)}=\omega$ and by fixing the ambiguity according to (38), the solutions for the adiabatic functions $F^{(n)}, G^{(n)}$ and $\omega^{(n)}$ are univocally determined. In Appendix $\mathrm{C}$ we give the four first terms of the adiabatic expansion.

\section{4D DIRAC FIELDS: ADIABATIC REGULARIZATION/RENORMALIZATION}

In this section we will carry out the detailed renormalization of the vacuum expectation value of the electric current $\left\langle j^{\mu}\right\rangle=-q\left\langle\bar{\psi} \gamma^{\mu} \psi\right\rangle$, which constitutes the most important physical quantity in the context of strong electrodynamics [44]. The only non-vanishing component of the electric current is the one parallel to the electric field. With the results of Sec. III A we can obtain the formal expression of the $z$-component of the mean electric current

$$
\begin{aligned}
\left\langle j^{3}\right\rangle & =\frac{2 q}{(2 \pi)^{3}} \int d^{3} k\left(\left|h_{\vec{k}}^{I I}\right|^{2}-\left|h_{\vec{k}}^{I}\right|^{2}\right) \\
& =\frac{q}{2 \pi^{2}} \int_{0}^{\infty} k_{\perp} d k_{\perp} \int_{-\infty}^{\infty} d k_{3}\left(\left|h_{\vec{k}}^{I I}\right|^{2}-\left|h_{\vec{k}}^{I}\right|^{2}\right),
\end{aligned}
$$

where $k_{\perp}=\sqrt{k_{1}^{2}+k_{2}^{2}}$. This expression is UV divergent and we have to renormalize it. The current has scaling dimension 3, meaning that the divergences could appear up to third adiabatic order, so we have to perform adiabatic subtractions until and including the third order (note that the energy-momentum tensor requires adiabatic subtractions of order 4) [8]. Therefore, the renormalized form of the electric current is

$\left\langle j^{3}\right\rangle_{\text {ren }}=\frac{q}{2 \pi^{2}} \int_{0}^{\infty} k_{\perp} d k_{\perp} \int_{-\infty}^{\infty} d k_{3}\left(\left|h_{\vec{k}}^{I I}\right|^{2}-\left|h_{\vec{k}}^{I}\right|^{2}-\left\langle j^{3}\right\rangle_{\vec{k}}^{(0-3)}\right)$

with $\left\langle j^{3}\right\rangle_{\vec{k}}^{(n)}=\left(\left|h_{\vec{k}}^{I I}\right|^{2}-\left|h_{\vec{k}}^{I}\right|^{2}\right)^{(n)}=-\frac{\omega-k_{3}}{2 \omega} \sum_{i=0}^{n} F^{(i)} F^{*(n-i)}+$ $\frac{\omega+k_{3}}{2 \omega} \sum_{i=0}^{n} G^{(i)} G^{*(n-i)}$. These subtraction terms contain all the divergences of the electric current, giving us a finite and meaningful result for $\left\langle j^{3}\right\rangle_{\text {ren }}$. The other components give a vanishing result. After computing the subtraction terms, we finally obtain

$$
\begin{aligned}
\left\langle j^{3}\right\rangle_{\text {ren }}= & \frac{q}{2 \pi^{2}} \int_{0}^{\infty} k_{\perp} d k_{\perp} \int_{-\infty}^{\infty} d k_{3}\left[\left(\left|h_{\vec{k}}^{I I}\right|^{2}-\left|h_{\vec{k}}^{I}\right|^{2}\right)-\frac{k_{3}}{\omega}\right. \\
& \left.-\frac{\kappa^{2} q A}{\omega^{3}}+\frac{3 \kappa^{2} k_{3} q^{2} A^{2}}{2 \omega^{5}}+\frac{\left(\kappa^{2}-4 k_{3}^{2}\right) \kappa^{2} q^{3} A^{3}}{2 \omega^{7}}+\frac{\kappa^{2} q \ddot{A}}{4 \omega^{5}}\right] .
\end{aligned}
$$

\section{A. Conformal anomaly}

An important test of any proposed renormalization method is the necessary agreement with the conformal anomaly. Here we compute the trace anomaly with our proposed extended adiabatic method. The trace of the energy-momentum tensor is proportional to the mass of the field $\left\langle T_{\mu}^{\mu}\right\rangle=m\langle\bar{\psi} \psi\rangle$. Although the two point function has to be renormalized until the third adiabatic order, the trace of the energy momentum tensor must be regularized up to fourth order, i.e.,

$$
\left\langle T_{\mu}^{\mu}\right\rangle_{\mathrm{ren}}=m\left(\langle\bar{\psi} \psi\rangle_{\mathrm{ren}}-\langle\bar{\psi} \psi\rangle^{(4)}\right) .
$$

In the massless limit the first term vanishes, so the anomaly should appear in the subtractions of adiabatic order 4 , that is

$$
\left\langle T_{\mu}^{\mu}\right\rangle_{\text {ren }}=-\lim _{m \rightarrow 0} m\langle\bar{\psi} \psi\rangle^{(4)}
$$

The vacuum expectation value of the two-point function $\langle\bar{\psi} \psi\rangle$ is given by

$$
\langle\bar{\psi} \psi\rangle=\frac{1}{2 \pi^{2}} \int_{0}^{\infty} k_{\perp} d k_{\perp} \int_{-\infty}^{\infty} d k_{3} \frac{m}{\kappa}\left(h_{\vec{k}}^{I *} h_{\vec{k}}^{I I}+h_{\vec{k}}^{I I *} h_{\vec{k}}^{I}\right) .
$$


By using the adiabatic regularization method, one can find the 4 th order subtraction terms. Hence, in the massless limit we get

$$
\begin{aligned}
\left\langle T_{\mu}^{\mu}\right\rangle_{\text {ren }} & =\lim _{m \rightarrow 0} \frac{m^{2}}{2 \pi^{2}} \int_{0}^{\infty} k_{\perp} d k_{\perp} \int_{-\infty}^{\infty} d k_{3} \frac{1}{\kappa}\left(h_{\vec{k}}^{I *} h_{\vec{k}}^{I I}+h_{\vec{k}}^{I I *} h_{\vec{k}}^{I}\right)^{(4)} \\
& =-\frac{q^{2} \dot{A}^{2}}{12 \pi^{2}} .
\end{aligned}
$$

One can easily rewrite this result in a covariant way, obtaining the result

$$
\left\langle T_{\mu}^{\mu}\right\rangle_{\mathrm{ren}}=\frac{q^{2}}{24 \pi^{2}} F_{\mu \nu} F^{\mu \nu} .
$$

It fully agrees with the well-known result for the trace anomaly induced by an electromagnetic field for a Dirac field [45].

\section{B. Relation with the DeWitt coefficients}

We will briefly see that the proposed adiabatic expansion for the fermionic modes agrees with the Schwinger-DeWitt adiabatic expansion for the Feynman propagator. We have proved this for the adiabatic expansion of the twodimensional theory in Sec. II. In the previous subsection we have implicitly obtained the 4th adiabatic order, given by

$$
\langle\bar{\psi} \psi\rangle^{(4)}=-\frac{1}{16 \pi^{2} m}\left(\frac{2}{3} q^{2} F_{\mu \nu} F^{\mu \nu}\right)=-\frac{\operatorname{tr} E_{2}}{16 \pi^{2} m}
$$

where $E_{2}$ coincides with the corresponding DeWitt coefficient at coincidence. Note that the numerical coefficient in the denominator is $(4 \pi)^{d / 2}$, where $d$ is the spacetime dimension. Moreover, at 6th adiabatic order we obtain

$$
\langle\bar{\psi} \psi\rangle^{(6)}=-\frac{1}{16 \pi^{2} m}\left(\frac{2 q^{2} \ddot{A}^{2}}{15 a^{2}}+\frac{2 q^{2} A^{(3)} \dot{A}}{5 a^{2}}\right)
$$

We can rewrite the above expression in a covariant form. It can be checked that it also fits with the DeWitt coefficient $E_{3}$

$$
\langle\bar{\psi} \psi\rangle^{(6)}=-\frac{\operatorname{tr} E_{3}}{16 \pi^{2} m^{3}}
$$

The general expression for $E_{3}$ is given in Appendix A. Here only the flat space terms are relevant

$$
\begin{aligned}
E_{3}= & -\frac{1}{360}\left(8 W_{\mu \nu ; \rho} W^{\mu \nu ; \rho}+2 W_{\mu \nu}{ }^{i \nu} W_{; \rho}{ }^{\mu \rho}+12 W_{\mu \nu ; \rho}{ }^{\rho} W^{\mu \nu}\right. \\
& -12 W_{\mu \nu} W^{\nu \rho} W_{\rho}{ }^{\mu}+6 Q_{; \mu}{ }^{\mu}{ }_{\nu}{ }^{\nu}+60 Q Q_{; \mu}{ }^{\mu}+30 Q_{; \mu} Q^{; \mu} \\
& \left.+60 Q^{3}+30 Q W_{\mu \nu} W^{\mu \nu}\right)
\end{aligned}
$$

where $Q=-\frac{i}{2} q F_{\mu \nu} \underline{\gamma}^{\mu} \underline{\gamma}^{\nu}$ and $W_{\mu \nu}=-i q F_{\mu \nu} I$. We reinforce that the adiabatic order assignment 1 for $A_{\mu}$ is a basic ingredient for achieving the above equivalence.

\section{Introduction of a mass scale and renormalization ambiguities}

A crucial point in the adiabatic regularization method is to fix the leading order of the adiabatic expansion, namely $\omega^{(0)}$. It seems very natural to define it as $\omega^{(0)} \equiv \omega=\sqrt{\vec{k}^{2}+m^{2}}$, as we did in Sec. III A. However, there exist an inherent ambiguity in the method [46]. It is possible to choose a slightly different expression for the leading term $\omega^{(0)} \equiv \omega_{\mu}=\sqrt{\vec{k}^{2}+\mu^{2}}$, where $\mu$ corresponds to an arbitrary mass scale. In order to obtain the new adiabatic subtractions with this new choice of the leading order, one has to rewrite the mode equations as

$$
\begin{aligned}
i \partial_{t} h_{\vec{k}}^{I} & =-\left(k_{3}+q A(t)\right) h_{\vec{k}}^{I}-\left(\kappa_{\mu}+\sigma\right) h_{\vec{k}}^{I I} \\
i \partial_{t} h_{\vec{k}}^{I I} & =\left(k_{3}+q A(t)\right) h_{\vec{k}}^{I I}-\left(\kappa_{\mu}+\sigma\right) h_{\vec{k}}^{I}
\end{aligned}
$$

where $\sigma=\kappa-\kappa_{\mu} \equiv \sqrt{k_{1}^{2}+k_{2}^{2}+m^{2}}-\sqrt{k_{1}^{2}+k_{2}^{2}+\mu^{2}}$ is assumed of adiabatic order 1 . Note that we recover the original adiabatic subtraction method by choosing $\mu=m$, and hence $\sigma=0$.

In this context, the ansatz of the adiabatic expansion will take the form

$$
\begin{aligned}
h_{\vec{k}}^{I} & =\sqrt{\frac{\omega_{\mu}-k_{3}}{2 \omega_{\mu}}} F_{\mu}(t) e^{-i \int^{t} \Omega_{\mu}\left(t^{\prime}\right) d t^{\prime}}, \\
h_{\vec{k}}^{I I} & =-\sqrt{\frac{\omega_{\mu}+k_{3}}{2 \omega_{\mu}}} G_{\mu}(t) e^{-i \int^{t} \Omega_{\mu}\left(t^{\prime}\right) d t^{\prime}},
\end{aligned}
$$

where the functions $F_{\mu}(t), G_{\mu}(t)$ and $\Omega_{\mu}(t)$ are expanded adiabatically as in (31). In order to recover at order 0 the limit of vanishing electric field (and also the limit $\sigma \rightarrow 0$, since $\sigma$ is now assumed of adiabatic order 1) we demand as initial conditions $F_{\mu}^{(0)}=1, G_{\mu}^{(0)}=1$ and $\omega_{\mu}^{(0)}=\omega_{\mu}$. With this new choice we can obtain the expressions of the adiabatic terms $\omega_{\mu}^{(n)}, F_{\mu}^{(n)}$ and $G_{\mu}^{(n)}$ as before: introducing the ansatz (52) in the mode equations (51) and in the normalization condition (26), expanding the functions $F_{\mu}(t), G_{\mu}(t)$ and $\Omega_{\mu}(t)$ adiabatically, and finally, solving them recursively, order by order. In Appendix D we give the details of the computation and also the expression of the adiabatic renormalization subtractions for the electric current. We remark that the introduction of a mass scale $\mu$ causes an unavoidable ambiguity in the renormalization procedure: it allows us to perform different adiabatic subtractions to render finite the physical observables, depending on the scale $\mu$ we choose. For instance, concerning the renormalized current $\left\langle\bar{\psi} \gamma^{\nu} \psi\right\rangle$ one can compare it at two different scales. Using the results given in the Appendix D we easily obtain 
$\left\langle\bar{\psi} \gamma^{\nu} \psi\right\rangle_{\text {ren }}(\mu)-\left\langle\bar{\psi} \gamma^{\nu} \psi\right\rangle_{\text {ren }}\left(\mu_{0}\right)=\frac{q}{12 \pi^{2}} \ln \left(\frac{\mu^{2}}{\mu_{0}^{2}}\right) \nabla_{\sigma} F^{\sigma \nu}$.

This ambiguity can be absorbed in the renormalization of the coupling constant. To this end it is convenient to scale the field as $\tilde{A}^{\nu} \equiv q A^{\nu}$ and rewrite the semiclassical Maxwell equations as

$$
\frac{1}{q^{2}} \nabla_{\alpha} \tilde{F}^{\alpha \beta}=-\left\langle\bar{\psi} \gamma^{\nu} \psi\right\rangle_{\text {ren }}
$$

The above relation for the current (53), reexpressed in terms of $\tilde{F}^{\alpha \beta}$, translates into the well-known shift: $q^{-2}(\mu)-q^{-2}\left(\mu_{0}\right)=-\left(12 \pi^{2}\right)^{-1} \ln \frac{\mu^{2}}{\mu_{0}^{2}}$, obtained within perturbative QED using minimal subtraction in dimensional regularization [47]. The renormalized current given in (41) should be understood as defined at the natural scale of the problem, defined by the physical mass of the charged field, i.e., $\mu=m$ and hence $q \equiv q(m)$.

\section{PHYSICAL APPLICATION: THE SAUTER ELECTRIC PULSE}

As mentioned in the Introduction, one of the main advantages of the adiabatic renormalization method is its proficiency to perform numerical computations and analytical approximations. We will devote this section to study the properties of the renormalized expression of the current (41) for the case of a pulsed electric field in a $1+3$ dimensional setting.

Let us consider the well-known Sauter-type pulse $E(t)=$ $E_{0} \cosh ^{-2}(t / \tau)$ with $\tau>0$, and its corresponding potential $A(t)=-E_{0} \tau \tanh (t / \tau)$, which is bounded at early and late times, $A( \pm \infty)=\mp E_{0} \tau$. This kind of pulse produces a

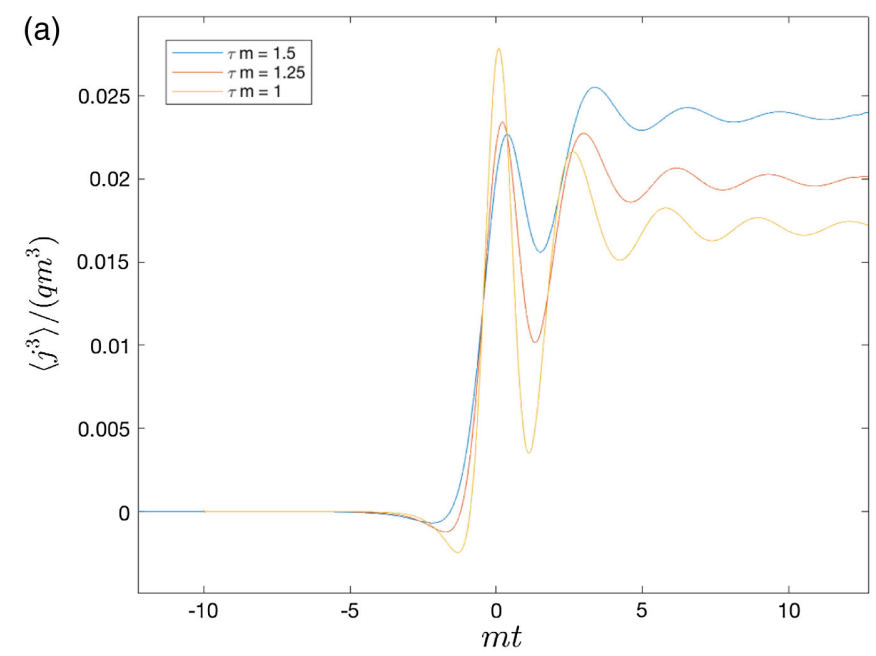

number of particles, and then also a current, which tends to be constant when $t \rightarrow \infty$. In Fig. 1 we represent the evolution of the current induced by this pulse for different values of $E_{0}$ and $\tau$. These figures have been obtained by solving numerically the differential equations for the modes and integrating the expression of the renormalized current (41).

\section{A. Late times behavior of the electric current}

We can obtain an expression of the current at late times for an electric background that vanishes at early and late times. Let us consider a pulse such that in the early and late time limits the potential is bounded as $A(-\infty)=-A_{0}$, $A(\infty)=A_{0}$, and its derivatives vanish. From Eqs. (22) and (23), one can see that at late times $t \rightarrow+\infty$ the modes behave as [31]

$$
\begin{aligned}
h_{\vec{k}}^{I / I I}(t) \sim & \pm \sqrt{\frac{\omega_{\text {out }} \mp\left(k_{3}+q A_{0}\right)}{2 \omega_{\text {out }}}} \alpha_{\vec{k}} e^{-i \omega_{\text {out }} t} \\
& +\sqrt{\frac{\omega_{\text {out }} \pm\left(k_{3}+q A_{0}\right)}{2 \omega_{\text {out }}}} \beta_{\vec{k}} e^{i \omega_{\text {out }} t},
\end{aligned}
$$

where $\omega_{\text {in/out }}=\sqrt{\left(k_{3} \mp q A_{0}\right)^{2}+\kappa^{2}}$, and $\alpha_{\vec{k}}$ and $\beta_{\vec{k}}$ are the usual Bogoliubov coefficients satisfying the relation $\left|\alpha_{\vec{k}}\right|^{2}+\left|\beta_{\vec{k}}\right|^{2}=1$, that ensures the normalization condition (26). The coefficient $\left|\beta_{\vec{k}}\right|^{2}$ gives the density number of created particles at any value of $\vec{k}$.

The renormalized electric current at late times induced by an electric pulse in terms of the coefficient $\left|\beta_{\vec{k}}\right|^{2}$ can be obtained by introducing the expression of the modes at late

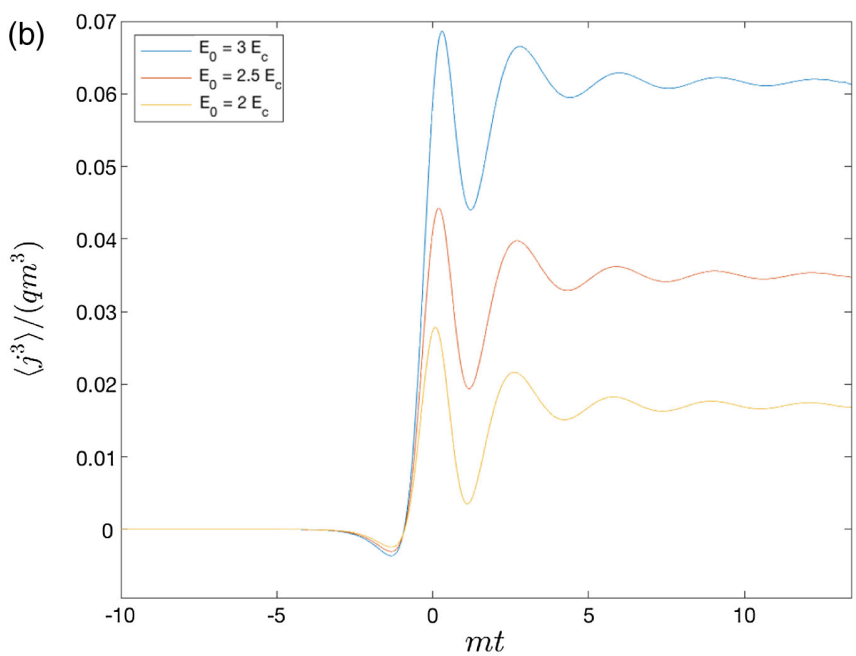

FIG. 1. Evolution of the renormalized current induced by a Sauter-type electric pulse for different values of the parameters. In figure (a) the field strength is fixed $\left(E_{0}=2 E_{c}\right.$ ), where $E_{c}=m^{2} / q$ is the critical electric field (or Schwinger limit), that is the scale above which the electric field can produce particles. In figure (b) the width of the pulse is fixed $(\tau=1 / \mathrm{m})$. We have used dimensionless variables, in terms of the mass and the charge. 
times (55) in the expression of the current (41). We obtain, for large $t$,

$$
\begin{aligned}
\left\langle j^{3}\right\rangle_{\text {ren }} \sim & -\frac{q}{\pi^{2}} \int_{0}^{\infty} k_{\perp} d k_{\perp} \int_{-\infty}^{\infty} d k_{3} \frac{k_{3}+q A_{0}}{\omega_{\text {out }}}\left|\beta_{\vec{k}}\right|^{2} \\
& +\frac{q}{2 \pi^{2}} \int_{0}^{\infty} k_{\perp} d k_{\perp} \int_{-\infty}^{\infty} d k_{3}\left[\frac{k_{3}+q A_{0}}{\omega_{\text {out }}}-\frac{k_{3}}{\omega}\right. \\
& \left.-\frac{\kappa^{2} q A_{0}}{\omega^{3}}+\frac{3 \kappa^{2} k_{3} q^{2} A_{0}^{2}}{2 \omega^{5}}+\frac{\left(\kappa^{2}-4 k_{3}^{2}\right) \kappa^{2} q^{3} A_{0}^{3}}{2 \omega^{7}}\right] .
\end{aligned}
$$

In Appendix E we prove that the second integral of this expression vanishes, so the current at late times is given by the simple expression

$\left\langle j^{3}\right\rangle_{\text {ren }} \sim-\frac{q}{\pi^{2}} \int_{0}^{\infty} k_{\perp} d k_{\perp} \int_{-\infty}^{\infty} d k_{3} \frac{k_{3}+q A_{0}}{\omega_{\text {out }}}\left|\beta_{\vec{k}}\right|^{2}$.

As expected, the final current is related to the number density of particles. The analytic expression of $\left|\beta_{\vec{k}}\right|^{2}$ depends on the form of the background.

\section{B. Scaling behavior for large field strength}

It is interesting to study the behavior of the current in the limit of large field strength. To this end, we consider again the example of the Sauter pulse, for which the coefficient $\left|\beta_{\vec{k}}\right|^{2}$ is given by (see [31] for more details)

$$
\left|\beta_{\vec{k}}\right|^{2}=\frac{\cosh \left(2 \pi q E_{0} \tau^{2}\right)-\cosh \left(\pi\left(\omega_{\text {out }}-\omega_{\text {in }}\right) \tau\right)}{2 \sinh \left(\pi \omega_{\text {in }} \tau\right) \sinh \left(\pi \omega_{\text {out }} \tau\right)} .
$$

Plugging it into (57) we can obtain the current at late times induced by the pulse. As a test, one can compare the results given by (57) with the ones given by the exact expression (41) for large $t$, which are represented in Fig. 1.

For this pulse, assuming $q E_{0}>0$, the large field strength limit corresponds to $q E_{0} \gg 0$. A numerical analysis of the expression (58) shows that the relevant values of $\kappa$ and $k_{3}$ are of the order of $\sqrt{q E_{0}}$ and $q E_{0} \tau$, respectively. Therefore, in order to study properly the limit of large $E_{0}$, it is convenient to introduce the following set of dimensionless variables

$$
\tilde{k}_{3}=\frac{k_{3}}{q E_{0} \tau}, \quad \tilde{\kappa}=\frac{\kappa}{\sqrt{q E_{0}}}, \quad x=q E_{0} \tau^{2},
$$

and study the limit $x \rightarrow \infty$ maintaining $\tilde{k}_{3}$ and $\tilde{\kappa}$ constant. Then, we rewrite $\left|\beta_{\vec{k}}\right|^{2}$ as

$$
\left|\beta_{\vec{k}}\right|^{2}=\frac{\cosh (2 \pi x)-\cosh \left(\pi\left(\tilde{\omega}_{\text {out }}(x)-\tilde{\omega}_{\text {in }}(x)\right)\right)}{2 \sinh \left(\pi \tilde{\omega}_{\text {out }}(x)\right) \sinh \left(\pi \tilde{\omega}_{\text {in }}(x)\right)},
$$

where $\tilde{\omega}_{\text {in/out }}(x)=\sqrt{x^{2}\left(\tilde{k}_{3} \pm 1\right)^{2}+x \tilde{\kappa}^{2}}$. In the limit $x \rightarrow \infty$ the above expression for $\left|\beta_{\vec{k}}\right|^{2}$ is independent of $x$, and it is given by

$$
\begin{aligned}
\left|\beta_{\vec{k}}\right|^{2} & \sim e^{-\pi \frac{\hat{\kappa}^{2}}{1-\tilde{k}_{3}^{2}} \Theta\left(1-\left|\tilde{k}_{3}\right|\right)} \\
& =e^{-\pi \frac{k_{\perp}^{2}+m^{2}}{q E_{0}}\left(\frac{1}{1-\left(\frac{k_{3}}{q E_{0} \tau^{2}} \tau^{2}\right.}\right)} \Theta\left(q E_{0} \tau-\left|k_{3}\right|\right) .
\end{aligned}
$$

Substituting the expression (61) into (57) and taking into account that $\frac{k_{3}-q E_{0} \tau}{\omega_{\text {out }}} \sim-1$ for large $E_{0}$, we obtain the behavior of the current at late times created by a high intensity pulse

$$
\left\langle j^{3}\right\rangle_{\mathrm{ren}} \sim \frac{q^{3} E_{0}^{2} \tau}{2 \pi^{3}} \int_{-1}^{1} d s\left(1-s^{2}\right) e^{-\pi \frac{m^{2}}{q E_{0}}\left(\frac{1}{1-s^{2}}\right)} .
$$

Assuming now that $q E_{0} \gg m^{2}$, the above integral (62) can be done exactly and we finally obtain

$$
\left\langle j^{3}\right\rangle_{\text {ren }} \sim \frac{2}{3 \pi^{3}} q^{3} E_{0}^{2} \tau,
$$

which is the predicted expression of the current in the limit of large field strength $E_{0}$. We can also obtain the total number density of created quanta for the Sauter pulse in this limit

$$
\langle N\rangle=\sum_{\lambda} \int \frac{d^{3} k}{(2 \pi)^{3}}\left(\left|\beta_{\vec{k}}\right|^{2}+\left|\beta_{-\vec{k}}\right|^{2}\right) \sim \frac{2}{3 \pi^{3}} q^{2} E_{0}^{2} \tau .
$$

It is interesting to compare the result (63) with the one obtained for a scalar field. The coefficient $\left|\beta_{\vec{k}}\right|^{2}$ in this case has a different expression, but it tends to the same limit for large $E_{0}$ (61). Therefore the scaling behavior of the current at late times $\left(\left\langle j^{3}\right\rangle_{\text {ren }}^{\text {scalar }} \sim \frac{1}{3 \pi^{3}} q^{3} E_{0}^{2} \tau\right)$ will be the same as in the fermionic case, except for the factor 2, on account of the absence of the spin degree of freedom.

For completeness, it is worth to see how the above results can also serve to describe the Schwinger limit, i.e, a constant electric field. Note that the expression (61) has been obtained for the limit $E_{0} \tau^{2} \gg 0$, so it should also be valid for the limit of large $\tau$, keeping $E_{0}$ constant, which describes a pulse with a large width. Bringing this limit to the extreme case $\tau \rightarrow \infty$, we get $\left|\beta_{\vec{k}}\right|^{2} \sim \exp \left(-\pi \frac{k_{\perp}^{2}+m^{2}}{q E_{0}}\right)$, which is the well-known expression for the beta coefficients of a constant electric field [12] leading to the Schwinger formula for the vacuum persistence amplitude.

\section{CONCLUSIONS}

In this work we have extended the adiabatic regularization method for 4-dimensional Dirac fields interacting with a time-varying electric background. Our approach can be distinguished from previous analysis in the literature in the adiabatic order assignment for the vector potential, which is chosen to be of order 1 . This choice is required to fit it with the expected equivalence with the 
Schwinger-DeWitt adiabatic expansion. Our proposal has required to introduce a nontrivial ansatz, Eq. (30), to generate a self-consistent adiabatic expansion of the fermionic modes. The given expansion turns out to be different from the WKB-type expansion used for scalar fields. With this extension we have obtained a well-defined prediction, Eq. (41), for the renormalized electric current induced by the created particles. Our proposal is consistent, in the massless limit, with the conformal anomaly. The expected equivalence with the Schwinger-DeWitt expansion is explicitly realized. In parallel we have also explored the physical consequences of the introduction of an arbitrary mass scale on the adiabatic regularization scheme, finding consistency with the behavior of the effective scaling of the electric coupling constant. To illustrate the power of the method we have analyzed the pair production phenomenon in the particular case of a Sauter-type electric pulse.
In particular, we have obtained the scaling behavior of the current in the strong field regime [Eq. (63)].

\section{ACKNOWLEDGMENTS}

We thank I. Agullo, P. R. Anderson, A. del Rio and A. Ferreiro for very useful comments. This work was supported by the Spanish MINECO research Grants No. FIS2017-84440-C2-1-P and No. FIS2017-91161EXP, and by the Generalitat Valenciana Grant No. SEJI/ 2017/042. P. B. is supported by the Formación del Personal Universitario Ph.D. fellowship No. FPU17/03712. S. P. is supported by the Formación del Personal Universitario Ph.D. fellowship No. FPU16/05287.

\section{APPENDIX A: DEWITT COEFFICIENT $\boldsymbol{E}_{3}$}

The expression for the DeWitt coefficient of sixth adiabatic order is $[42,43]$

$$
\begin{aligned}
& E_{3}=-\frac{1}{7 !}\left(-18 R_{; \mu}{ }^{\mu \nu}{ }_{\nu}+17 R_{; \mu} R^{; \mu}-2 R_{\mu \nu ; \rho} R^{\mu \nu ; \rho}-4 R_{\mu \nu ; \rho} R^{\mu \rho ; \nu}+9 R_{\mu \nu \rho \sigma ; \alpha} R^{\mu \nu \rho \sigma ; \alpha}+28 R R_{; \mu}{ }^{\mu}-8 R_{\mu \nu} R_{; \rho}^{\mu \nu} \rho\right. \\
& +24 R_{\mu \nu} R_{\rho}^{\mu \rho ; \nu}+12 R_{\mu \nu \rho \sigma} R_{\alpha}^{\mu \nu \rho \sigma ; \alpha}-\frac{35}{9} R^{3}+\frac{14}{3} R R_{\mu \nu} R^{\mu \nu}-\frac{14}{3} R R_{\mu \nu \rho \sigma} R^{\mu \nu \rho \sigma}+\frac{208}{9} R_{\mu \nu} R^{\mu \rho} R_{\rho}^{\nu} \\
& \left.-\frac{64}{3} R_{\mu \nu} R_{\rho \sigma} R^{\mu \rho \nu \sigma}+\frac{16}{3} R_{\mu}{ }^{\nu} R^{\mu \rho \sigma \alpha} R_{\nu \rho \sigma \alpha}-\frac{44}{9} R_{\mu \nu \rho \sigma} R^{\mu \nu \alpha \beta} R_{\alpha \beta}^{\rho \sigma}-\frac{80}{9} R_{\mu \nu \rho \sigma} R^{\mu \alpha \rho \beta} R_{\alpha \beta}^{\nu}{ }_{\alpha}\right) I \\
& -\frac{1}{360}\left(8 W_{\mu \nu ; \rho} W^{\mu \nu ; \rho}+2 W_{\mu \nu}^{; \nu} W_{; \rho}^{\mu \rho}+12 W_{\mu \nu ; \rho}{ }^{\rho} W^{\mu \nu}-12 W_{\mu \nu} W^{\nu \rho} W_{\rho}^{\mu}-6 R_{\mu \nu \rho \sigma} W^{\mu \nu} W^{\rho \sigma}+4 R_{\mu \nu} W^{\mu \rho} W_{\rho}^{\nu}\right. \\
& -5 R W_{\mu \nu} W^{\mu \nu}+6 Q_{; \mu^{\mu}}{ }_{\nu}{ }^{\nu}+60 Q Q_{; \mu}{ }^{\mu}+30 Q_{; \mu} Q^{; \mu}+60 Q^{3}+30 Q W_{\mu \nu} W^{\mu \nu}-10 R Q_{; \mu}{ }^{\mu}-4 R_{\mu \nu} Q^{; \mu \nu}-12 R_{; \mu} Q^{; \mu} \\
& \left.-30 Q^{2} R-12 Q R_{; \mu}{ }^{\mu}+5 Q R^{2}-2 Q R_{\mu \nu} R^{\mu \nu}+2 Q R_{\mu \nu \rho \sigma} R^{\mu \nu \rho \sigma}\right)
\end{aligned}
$$

where, for scalar fields $Q=\xi R, W_{\mu \nu}=i q F_{\mu \nu}$ and $I=1$, while for Dirac fields $Q=\frac{1}{4} R I-\frac{i}{2} q F_{\mu \nu} \underline{\gamma}^{\mu} \underline{\gamma}^{\nu}, W_{\mu \nu}=$ $-i q F_{\mu \nu} I-\frac{1}{4} R_{\mu \nu \rho \sigma} \underline{\gamma}^{\rho} \underline{\gamma}^{\sigma}$ and $I$ is the identity matrix.

\section{APPENDIX B: MATCHING HADAMARD COEFFICIENTS WITH SCALAR ADIABATIC REGULARIZATION}

In this appendix, we relate the adiabatic regularization method with Hadamard renormalization for charged scalar fields. To simplify the comparison we will restrict the analysis to Minkowski spacetime. In Sec. II we have introduced the basics of the adiabatic regularization method for 4-dimensional charged scalar fields interacting with an electromagnetic background. The renormalized vacuum expectation value on the two point function was given in (4). For the electric current, defined as $j_{\mu}=i q\left[\phi^{\dagger} D^{\mu} \phi-\left(D^{\mu} \phi\right)^{\dagger} \phi\right]$, we obtain $\left\langle j^{3}\right\rangle_{\text {ren }}=\frac{q}{(2 \pi)^{3}} \int d^{3} k\left[\left(k_{3}-q A\right)\left|h_{\vec{k}}\right|^{2}-\left\langle j^{3}\right\rangle_{\vec{k}}^{(0-3)}\right]$, with $\left\langle j^{3}\right\rangle_{\vec{k}}^{(0-3)}=\sum_{n=0}^{3} k_{3}\left(\Omega_{\vec{k}}^{-1}\right)^{(n)}-q A\left\langle\phi^{\dagger} \phi\right\rangle_{\vec{k}}^{(0-2)}$. To compute these subtraction terms, we usually fix the leading order of the adiabatic expansion as $\omega^{(0)}=\omega=\sqrt{\vec{k}^{2}+m^{2}}$.

As explained in the main text, the choice of the leading term is crucial to define the adiabatic expansion. We have argued that to properly fix the leading therm we have to choose the vector potential $A$ of adiabatic order 1 . However, the choice of the leading term $\omega^{(0)}$ is not yet completely fixed, and one can make a more general choice defining $\omega^{(0)}=\omega_{\mu}=\sqrt{\vec{k}^{2}+\mu^{2}}$, where $\mu$ is an arbitrary mass scale. With this new choice the adiabatic expansion can be recalculated, giving us slightly different subtraction terms. An exhaustive analysis of this ambiguity can be found in 
[46]. The ambiguity on the subtractions, leads to an ambiguity on the physical observables. For the two point function the ambiguity manifests as

$\left\langle\phi^{\dagger} \phi\right\rangle_{\text {ren }}(\mu)=\left\langle\phi^{\dagger} \phi\right\rangle_{\text {ren }}\left(\mu_{0}\right)-\frac{\alpha}{2}\left[m^{2} \ln \left(\frac{\mu^{2}}{\mu_{0}^{2}}\right)-\mu^{2}+\mu_{0}^{2}\right]$,

where $\alpha=\frac{1}{2(2 \pi)^{2}}$, and for the electric current we find

$$
\left\langle j^{3}\right\rangle_{\text {ren }}(\mu)=\left\langle j^{3}\right\rangle_{\text {ren }}\left(\mu_{0}\right)-\frac{\alpha}{6} \ln \left(\frac{\mu^{2}}{\mu_{0}^{2}}\right) q^{2} \ddot{A} .
$$

Rewriting the equation above in a covariant way, we get

$$
\left\langle j^{\nu}\right\rangle_{\text {ren }}(\mu)=\left\langle j^{\nu}\right\rangle_{\text {ren }}\left(\mu_{0}\right)-\frac{q^{2} \alpha}{6} \ln \left(\frac{\mu^{2}}{\mu_{0}^{2}}\right) \nabla_{\sigma} F^{\sigma \nu} .
$$

\section{Matching with Hadamard renormalization}

We can compare the results summarized in Sec. II with the results given by Hadamard renormalization, particularizing for the case in which $A_{\mu}=(0,0,0,-A(t))$. Adopting the notation given in [39], the expectation value of the two point function can be expressed as

$$
\left\langle\phi \phi^{\dagger}\right\rangle_{\text {ren }}=\alpha w_{0}(x)
$$

and the electric current is given by

$$
\left\langle j_{\mu}\right\rangle=-2 q \alpha\left(q A_{\mu} w_{0}(x)+\Im\left[w_{1 \mu}(x)\right]\right),
$$

where $\alpha=\frac{1}{2(2 \pi)^{2}}$ and the functions $w_{0}$ and $w_{1 \mu}$ are the first terms of the covariant Taylor series expansion of the Hadamard biscalar $W\left(x, x^{\prime}\right)$.

Comparing (B5) with (4) we immediately get

$$
\alpha w_{0}=\frac{1}{2(2 \pi)^{3}} \int d^{3} k\left[\left|h_{\vec{k}}\right|^{2}-\sum_{n=0}^{2}\left(\Omega_{\vec{k}}^{-1}\right)^{(n)}\right],
$$

and hence, by using the previous result and Eqs. (B1) and (B6) we directly find

$$
\alpha \Im\left(w_{13}\right)=\frac{q}{2(2 \pi)^{3}} \int d^{3} k\left[k_{3}\left|h_{\vec{k}}\right|^{2}-\sum_{n=0}^{3} k_{3}\left(\Omega_{\vec{k}}^{-1}\right)^{(n)}\right] .
$$

Hadamard renormalization scheme also presents a renormalization ambiguity in even space-time dimensions, due to the choice of the renormalization lenght scale $\ell$. The ambiguity is manifested in the physical observables as

$$
\left\langle\phi \phi^{\dagger}\right\rangle_{\text {ren }} \rightarrow\left\langle\phi \phi^{\dagger}\right\rangle_{\text {ren }}+\frac{\alpha}{2} m^{2} \ln \ell^{2},
$$

$$
\left\langle j_{\mu}\right\rangle_{\text {ren }} \rightarrow\left\langle j_{\mu}\right\rangle_{\text {ren }}+\frac{\alpha q^{2}}{6}\left(\nabla^{\rho} F_{\rho \mu}\right) \ln \ell^{2} .
$$

Note that the length scale $\ell$ is inversely proportional to the mass scale $\mu$. Comparing these results with the ones obtained with adiabatic regularization [Eqs. (B2) and (B4)] we find that the logarithmic part of the ambiguity is exactly the same. However, with adiabatic regularization we also find a quadratic term in the ambiguity of the two point function.

\section{APPENDIX C: SUBTRACTION TERMS}

In this appendix we give the explicit expressions of the adiabatic expansion of the fermionic field modes up to and including the fourth adiabatic order. We remind that $G^{(n)}\left(k_{3}, q A\right)=F^{(n)}\left(-k_{3},-q A\right)$.

Order 0

$$
\omega^{(0)}=\omega, \quad F_{x}^{(0)}=G_{x}^{(0)}=1, \quad F_{y}^{(0)}=G_{y}^{(0)}=0 .
$$

Order 1

$\omega^{(1)}=\frac{q A k_{3}}{\omega}, \quad F_{x}^{(1)}=-\frac{q A\left(\omega+k_{3}\right)}{2 \omega^{2}}, \quad F_{y}^{(1)}=G_{y}^{(1)}=0$.

Order 2

$\omega^{(2)}=\frac{q^{2} A^{2} \kappa^{2}}{2 \omega^{4}}, \quad F_{x}^{(2)}=-\frac{5 q^{2} A^{2} \kappa^{2}}{8 \omega^{4}}+\frac{q^{2} A^{2}\left(\omega+k_{3}\right)}{2 \omega^{3}}$,

$F_{y}^{(2)}=-G_{y}^{(2)}=\frac{q \dot{A}}{4 \omega^{2}}$.

Order 3

$$
\begin{aligned}
\omega^{(3)}= & -\frac{q^{3} A^{3} \kappa^{2} k_{3}}{2 \omega^{5}}-\frac{q \ddot{A} k_{3}}{4 \omega^{3}}, \\
F_{x}^{(3)}= & \frac{11 q^{3} A^{3} \kappa^{2}}{16 \omega^{5}}-\frac{q^{3} A^{3}}{2 \omega^{3}}+\frac{15 q^{3} A^{3} \kappa^{2} k_{3}}{16 \omega^{6}} \\
& -\frac{q^{3} A^{3} k_{3}}{2 \omega^{4}}+\frac{q \ddot{A}\left(\omega+k_{3}\right)}{8 \omega^{4}}, \\
F_{y}^{(3)}= & -G_{y}^{(3)}=-\frac{5 q^{2} A \dot{A} k_{3}}{8 \omega^{4}} .
\end{aligned}
$$

Order 4

$$
\begin{aligned}
\omega^{(4)}= & -\frac{5 q^{4} A^{4} \kappa^{4}}{8 \omega^{7}}+\frac{q^{4} A^{4} \kappa^{2}}{2 \omega^{5}}-\frac{3 \kappa^{2} q^{2} A \ddot{A}}{4 \omega^{5}} \\
& +\frac{5 q^{2} A \ddot{A}}{8 \omega^{3}}+\frac{5 k_{3}^{2} q^{2} \dot{A}^{2}}{8 \omega^{5}},
\end{aligned}
$$




$$
\begin{aligned}
F_{x}^{(4)}= & -\frac{17 A^{4} \kappa^{2} k_{3} q^{4}}{16 \omega^{7}}+\frac{A^{4} k_{3} q^{4}}{2 \omega^{5}}+\frac{195 A^{4} \kappa^{4} q^{4}}{128 \omega^{8}}-\frac{31 A^{4} \kappa^{2} q^{4}}{16 \omega^{6}} \\
& +\frac{A^{4} q^{4}}{2 \omega^{4}}-\frac{A k_{3} q^{2} \ddot{A}}{2 \omega^{5}}-\frac{5 \dot{A}^{2} k_{3} q^{2}}{16 \omega^{5}}+\frac{9 A \kappa^{2} q^{2} \ddot{A}}{16 \omega^{6}} \\
& +\frac{5 \dot{A}^{2} \kappa^{2} q^{2}}{16 \omega^{6}}-\frac{A q^{2} \ddot{A}}{2 \omega^{4}}-\frac{11 \dot{A}^{2} q^{2}}{32 \omega^{4}}, \\
F_{y}^{(4)}= & -G_{y}^{(4)}=\frac{q^{3} A^{2} \dot{A}\left(34 \omega^{2}-45 \kappa^{2}\right)}{32 \omega^{6}}-\frac{q A^{(3)}}{16 \omega^{4}} .
\end{aligned}
$$

\section{APPENDIX D: $\mu$-PARAMETER ADIABATIC EXPANSION}

The general solution for $F_{\mu}^{(n)}, G_{\mu}^{(n)}$ and $\omega_{\mu}^{(n)}$ is given by

$$
\begin{aligned}
\omega_{\mu}^{(n)}= & \omega^{(n)}\left(\omega_{\mu}, \kappa_{\mu}, F_{\mu}, G_{\mu}\right)+\frac{\sigma \kappa_{\mu}}{\omega_{\mu}}\left[\left(G_{\mu}\right)_{x}^{(n-1)}+\left(F_{\mu}\right)_{x}^{(n-1)}\right] \\
& +\frac{\sigma^{2}}{2 \omega_{\mu}}\left[\left(G_{\mu}\right)_{x}^{(n-2)}+\left(F_{\mu}\right)_{x}^{(n-2)}\right],
\end{aligned}
$$

$$
\begin{aligned}
\left(F_{\mu}\right)_{x}^{(n)}= & F_{x}^{(n)}\left(\omega_{\mu}, \kappa_{\mu}, F_{\mu}, G_{\mu}\right) \\
& +\frac{1}{4 \omega_{\mu}^{2}}\left\{\frac{\omega_{\mu}+k_{3}}{\omega_{\mu}-k_{3}}\left[2 \sigma \kappa_{\mu}\left(G_{\mu}\right)_{x}^{(n-1)}+\sigma^{2}\left(G_{\mu}\right)_{x}^{(n-2)}\right]\right. \\
& \left.-2 \sigma \kappa_{\mu}\left(F_{\mu}\right)_{x}^{(n-1)}-\sigma^{2}\left(F_{\mu}\right)_{x}^{(n-2)}\right\},
\end{aligned}
$$$$
\left(F_{\mu}\right)_{y}^{(n)}=F_{y}^{(n)}\left(\omega_{\mu}, \kappa_{\mu}, F_{\mu}, G_{\mu}\right)
$$$$
+\frac{1}{\kappa_{\mu}^{2}}\left[2 \sigma \kappa_{\mu}\left(G_{\mu}\right)_{y}^{(n-1)}+\sigma^{2}\left(G_{\mu}\right)_{y}^{(n-2)}\right],
$$

where $\omega^{(n)} / F^{(n)} / G^{(n)}\left(\omega_{\mu}, \kappa_{\mu}, F_{\mu}, G_{\mu}\right)$ are given by the expressions (35), (36) and (37) with the changes $(\omega, \kappa, F, G) \rightarrow\left(\omega_{\mu}, \kappa_{\mu}, F_{\mu}, G_{\mu}\right)$. Note again that $G_{\mu}\left(k_{3}, q A\right)$ satisfies the same equations than $F_{\mu}\left(-k_{3},-q A\right)$, and hence $G_{\mu}^{(n)}\left(k_{3}, q A\right)=F_{\mu}^{(n)}\left(-k_{3},-q A\right)$. We also find an ambiguity in the imaginary part (D1). For simplicity we choose

$$
\begin{aligned}
\left(F_{\mu}\right)_{y}^{(n)}= & -\left(G_{\mu}\right)_{y}^{(n)} \\
= & -\frac{\left(\omega_{\mu}-k_{3}\right)}{2 \kappa_{\mu}^{2}}\left[\left(\dot{F}_{\mu}\right)_{x}^{(n-1)}+\sum_{i=1}^{n-1} \omega_{\mu}^{(n-i)}\left(F_{\mu}\right)_{y}^{(i)}\right. \\
& +q A\left(F_{\mu}\right)_{y}^{(n-1)} \\
& \left.-\frac{1}{\omega_{\mu}-k_{3}}\left(2 \sigma \kappa_{\mu}\left(G_{\mu}\right)_{y}^{(n-1)}+\sigma^{2}\left(G_{\mu}\right)_{y}^{(n-2)}\right)\right]
\end{aligned}
$$

With the initial conditions $\left(F_{\mu}\right)_{x}^{(0)}=\left(G_{\mu}\right)_{x}^{(0)}=1$, $\left(F_{\mu}\right)_{y}^{(0)}=\left(G_{\mu}\right)_{y}^{(0)}=0$ and $\omega_{\mu}^{(0)}=\omega_{\mu}$ and by fixing the ambiguity (D4), the solutions for the adiabatic functions $F_{\mu}^{(n)}, G_{\mu}^{(n)}$ and $\omega_{\mu}^{(n)}$ are univocally determined.

The renormalized electric current for an arbitrary mass scale is given by

$$
\begin{aligned}
\left\langle j^{3}\right\rangle_{\text {ren }}= & \frac{q}{2 \pi^{2}} \int_{0}^{\infty} k_{\perp} d k_{\perp} \int_{-\infty}^{\infty} d k_{3}\left[\left(\left|h_{\vec{k}}^{I I}\right|^{2}-\left|h_{\vec{k}}^{I}\right|^{2}\right)\right. \\
& \left.-\left\langle j^{3}\right\rangle_{\vec{k}}^{(0-3)}(\mu)\right],
\end{aligned}
$$

with

$$
\begin{aligned}
\left\langle j^{3}\right\rangle_{\vec{k}}^{(0)}(\mu)= & \frac{k_{3}}{\omega_{\mu}}, \\
\left\langle j^{3}\right\rangle_{\vec{k}}^{(1)}(\mu)= & \frac{\kappa_{\mu}^{2} q A}{\omega_{\mu}^{3}}-\frac{2 k_{3} \kappa_{\mu} \sigma}{\omega_{\mu}^{3}} \\
\left\langle j^{3}\right\rangle_{\vec{k}}^{(2)}(\mu)= & -\frac{3 \kappa^{2} k_{3} q^{2} A^{2}}{2 \omega_{\mu}^{5}}-\frac{2 q A \kappa_{\mu} \sigma\left(3 \kappa_{\mu}^{2}-2 \omega_{\mu}^{2}\right)}{\omega_{\mu}^{5}} \\
& +\frac{3 k_{3} \sigma^{2}\left(2 \kappa_{\mu}^{2}-\omega_{\mu}^{2}\right)}{\omega_{\mu}^{5}}, \\
\left\langle j^{3}\right\rangle_{\vec{k}}^{(3)}(\mu)= & \frac{\kappa_{\mu}^{2} q^{3} A^{3}\left(4 \omega_{\mu}^{2}-5 \kappa_{\mu}^{2}\right)}{2 \omega_{\mu}^{7}} \\
& -\frac{2 k_{3} \sigma^{3}\left(10 \kappa_{\mu}^{4}-9 \kappa_{\mu}^{2} \omega_{\mu}^{2}+\omega_{\mu}^{4}\right)}{\kappa \omega_{\mu}^{7}} \\
& +\frac{3 k_{3} q^{2} A^{2} \sigma \kappa_{\mu}\left(5 \kappa_{\mu}^{2}-2 \omega_{\mu}^{2}\right)}{\omega_{\mu}^{7}} \\
& +\frac{3 q A \sigma^{2}\left(10 \kappa_{\mu}^{4}-11 \kappa_{\mu}^{2} \omega_{\mu}^{2}+2 \omega_{\mu}^{4}\right)}{\omega_{\mu}^{7}}-\frac{\kappa_{\mu}^{2} q \ddot{A}}{4 \omega_{\mu}^{5}} .
\end{aligned}
$$

\section{APPENDIX E: SIMPLIFICATION OF THE EXPRESSION OF THE CURRENT AT LATE TIMES}

In this appendix we prove that the second integral in the expression of the current at late times [see Eq. (56)],

$$
\begin{aligned}
I= & \int_{0}^{\infty} k_{\perp} d k_{\perp} \int_{-\infty}^{\infty} d k_{3}\left[\frac{k_{3}+q A_{0}}{\omega_{\text {out }}}-\frac{k_{3}}{\omega}-\frac{\kappa^{2} q A_{0}}{\omega^{3}}\right. \\
& \left.+\frac{3 \kappa^{2} k_{3} q^{2} A_{0}^{2}}{2 \omega^{5}}+\frac{\left(\kappa^{2}-4 k_{3}^{2}\right) \kappa^{2} q^{3} A_{0}^{3}}{2 \omega^{7}}\right]
\end{aligned}
$$

vanishes. Taking into account the property $(1+2 x y+$ $\left.y^{2}\right)^{-1 / 2}=\sum_{n=0}^{\infty} P_{n}(-x) y^{n}$, where $P_{n}(x)$ are the Legendre polynomials, we can expand the first term of the integral around $A_{0}=0$ as follows 


$$
\frac{k_{3}+q A_{0}}{\omega_{\text {out }}}=\sum_{n=0}^{\infty} c_{n}(\vec{k})\left(q A_{0}\right)^{n},
$$

where

$$
\begin{aligned}
& c_{0}(\vec{k})=\frac{k_{3}}{\omega}, \\
& c_{n}(\vec{k})=\frac{1}{\omega^{n}}\left[P_{n-1}\left(-\frac{k_{3}}{\omega}\right)+\frac{k_{3}}{\omega} P_{n}\left(-\frac{k_{3}}{\omega}\right)\right] \text { for } n>0 .
\end{aligned}
$$

One can see that the first four terms of this expansion give exactly the rest of the terms of the integral (E1) (the subtraction terms) with a global change of sign. Therefore they are cancelled and the integral can be written as

$$
I=\int_{0}^{\infty} k_{\perp} d k_{\perp} \sum_{n=4}^{\infty}\left[\left(q A_{0}\right)^{n} \int_{-\infty}^{\infty} d k_{3} c_{n}(\vec{k})\right] .
$$

Under the change of variable $x=-k_{3} / \omega$, the integral in $k_{3}$ can be rewritten as

$$
\begin{aligned}
\int_{-\infty}^{\infty} d k_{3} c_{n}(\vec{k})= & \frac{1}{\kappa^{n-1}}\left(\int_{-1}^{1} d x\left(1-x^{2}\right)^{\frac{n-3}{2}} P_{n-1}(x)\right. \\
& \left.-\int_{-1}^{1} d x x\left(1-x^{2}\right)^{\frac{n-3}{2}} P_{n}(x)\right) .
\end{aligned}
$$

The Legendre polynomials satisfy the property $P_{n}(-x)=$ $(-1)^{n} P_{n}(x)$, so it is trivial to see that for any even $n$ these integrals vanish. For odd values of $n$ and $n \geq 3$ the function $\left(1-x^{2}\right)^{\frac{n-3}{2}}$ is a polynomial of order $n-3$. Using the property $\int_{-1}^{1} d x \operatorname{Pol}_{a}(x) P_{b}(x)=0$ for $a<b$, where $\operatorname{Pol}_{a}(x)$ is a polynomial of order $a$, we get that the integrals in (E4) vanish for $n \geq 3$. This last property can be easily proven taking into account that $P_{n}(x)$ form a basis, and any function can be expanded as $f(x)=$ $\sum_{b=0}^{\infty} c_{b} P_{b}(x)$ where $c_{b}=(b+1 / 2) \int_{-1}^{1} d x f(x) P_{b}(x)$, and if the function is a polynomial $f(x)=\operatorname{Pol}_{a}(x)$, for consistency $c_{b}=0$ for any $b>a$. Therefore, for all values of $n$ involved in (E3) the integral vanishes, and then $I=0$, as we wanted to prove.
[1] W. Heisenberg and H. Euler, Z. Phys. 98, 714 (1936).

[2] F. Sauter, Z. Phys. 69, 742 (1931).

[3] J. Schwinger, Phys. Rev. 82, 664 (1951).

[4] L. Parker, The creation of particles in an expanding universe, Ph.D. thesis, Harvard University, 1966; Phys. Rev. Lett. 21, 562 (1968); Phys. Rev. D 183, 1057 (1969); 3, 346 (1971).

[5] Y. B. Zeldovich, Pis'ma Zh. Eksp. Teor. Fiz. 12, 443 (1970); Y. B. Zeldovich and A. A. Starobinsky, Zh. Eksp. Teor. Fiz. 61, 2161 (1971) [Sov. Phys. JETP 34, 1159 (1972)].

[6] L. H. Ford and L. Parker, Phys. Rev. D 16, 1601 (1977).

[7] S. W. Hawking, Nature (London) 248, 30 (1974); Commun. Math. Phys. 43, 199 (1975).

[8] L. Parker and D. J. Toms, Quantum Field Theory in Curved Spacetime: Quantized Fields and Gravity (Cambridge University Press, Cambridge, England, 2009).

[9] R. M. Wald, Quantum Field Theory in Curved Space-time and Black Hole Thermodynamics (University of Chicago Press, Chicago, 1994).

[10] S. Fulling, Aspects of Quantum Field Theory in Curved Space-Time (Cambridge University Press, Cambridge, England, 1989).

[11] N. D. Birrell and P.C.W. Davies, Quantum Fields in Curved Space (Cambridge University Press, Cambridge, England, 1982).

[12] N. B. Narozhnyi and A. I. Nikishov, Yad. Fiz. 11, 1072 (1970) [Sov. J. Nucl. Phys. 11, 596 (1970)].

[13] E. Brezin and C. Itzykson, Phys. Rev. D 2, 1191 (1970).

[14] L. Parker and S. A. Fulling, Phys. Rev. D 9, 341 (1974); S. A. Fulling and L. Parker, Ann. Phys. (N.Y.) 87, 176 (1974).

[15] N. D. Birrell, Proc. R. Soc. B 361, 513 (1978).
[16] P. R. Anderson and L. Parker, Phys. Rev. D 36, 2963 (1987).

[17] I. Agullo, J. Navarro-Salas, G. J. Olmo, and L. Parker, Phys. Rev. Lett. 103, 061301 (2009); Phys. Rev. D 81, 043514 (2010); I. Agullo, W. Nelson, and A. Ashtekar, Phys. Rev. D 91, 064051 (2015).

[18] A. del Rio and J. Navarro-Salas, Phys. Rev. D 91, 064031 (2015).

[19] A. Landete, J. Navarro-Salas, and F. Torrenti, Phys. Rev. D 88, 061501 (2013); 89, 044030 (2014); A. del Rio, J. Navarro-Salas, and F. Torrenti, Phys. Rev. D 90, 084017 (2014); S. Ghosh, Phys. Rev. D 91, 124075 (2015); 93, 044032 (2016); A. del Rio, A. Ferreiro, J. Navarro-Salas, and F. Torrenti, Phys. Rev. D 95, 105003 (2017).

[20] J. F. Barbero G., A. Ferreiro, J. Navarro-Salas, and E. J. S. Villaseñor, Phys. Rev. D 98, 025016 (2018).

[21] The extreme light infrastructure (ELI) project, www .extreme-light-infrastructure.eu/.

[22] V. Yakimenko et al., Phys. Rev. Lett. 122, 190404 (2019).

[23] R. Ruffini, G. Vereshchagin, and S. Xue, Phys. Rep. 487, 1 (2010).

[24] S. P. Kim, arXiv:1905.13439.

[25] M. B. Fröb, J. Garriga, S. Kann, M. Sasaki, J. Soda, T. Tanaka, and A. Vilenkin, J. Cosmol. Astropart. Phys. 04 (2014) 009; T. Kobayashi and N. Afshordi, J. High Energy Phys. 10 (2014) 166; C. Stahl, E. Strobel, and S.-S. Xue, Phys. Rev. D 93, 025004 (2016).

[26] C. Stahl, Nucl. Phys. B939, 95 (2019).

[27] S. Shakeri, M. A. Gorji, and H. Firouzjahi, Phys. Rev. D 99, 103525 (2019). 
[28] G. V. Dunne, Int. J. Mod. Phys. A 27, 1260004 (2012); Eur. Phys. J. D 55, 327 (2009); B. S. Xie, Z. L. Lie, and S. Tang, Matter Radiat. Extremes 2, 225 (2017).

[29] R. Dabrowski and G. V. Dunne, Phys. Rev. D 94, 065005 (2016).

[30] L. J. Garay, A. García Martín-Caro, and M. Martín-Benito, J. High Energy Phys. 04 (2020) 120.

[31] P. Beltrán, A. Ferreiro, J. Navarro-Salas, and S. Pla, Phys. Rev. D 100, 085014 (2019).

[32] F. Cooper and E. Mottola, Phys. Rev. D 40, 456 (1989).

[33] Y. Kluger, J. M. Eisenberg, B. Svetitsky, F. Cooper, and E. Mottola, Phys. Rev. Lett. 67, 2427 (1991).

[34] Y. Kluger, J. M. Eisenberg, B. Svetitsky, F. Cooper, and E. Mottola, Phys. Rev. D 45, 4659 (1992).

[35] A. Ferreiro and J. Navarro-Salas, Phys. Rev. D 97, 125012 (2018).

[36] A. Ferreiro, J. Navarro-Salas, and S. Pla, Phys. Rev. D 98, 045015 (2018).
[37] A. Ferreiro, J. Navarro-Salas, and S. Pla, arXiv:1903.11425.

[38] P. Beltrán-Palau, J. Navarro-Salas, and S. Pla, Phys. Rev. D 99, 105008 (2019).

[39] V. Balakumar and E. Winstanley, Classical Quantum Gravity 37, 065004 (2020).

[40] R. Herman and W. A. Hiscock, Phys. Rev. D 53, 3285 (1996).

[41] B. S. DeWitt, Dynamical Theory of Groups and Fields (Gordon and Breach, New York, 1965).

[42] P. B. Gilkey, J. Diff. Geom. 10, 601 (1975).

[43] D. V. Vassilevich, Phys. Rep. 388, 279 (2003).

[44] W. Pittrich and H. Gies, Probing the Quantum Vacuum (Springer, Heidelberg, 2000).

[45] M. J. Duff, Classical Quantum Gravity 11, 1387 (1994).

[46] A. Ferreiro and J. Navarro-Salas, Phys. Lett. B 792, 81 (2019).

[47] M.E. Peskin and D. V. Schroeder, An Introduction to Quantum Field Theory (Addison-Wesley, Reading, MA, 1995). 